\title{
On the Topological Conjugacy of Brouwer Flows
}

\author{
Zbigniew Leśniak ${ }^{1}$
}

Received: 7 December 2016 / Published online: 11 November 2017

(C) The Author(s) 2017. This article is an open access publication

\begin{abstract}
We study the problem of topological conjugacy of Brouwer flows. We give a sufficient and necessary condition for Brouwer flows to be topologically conjugate. To obtain this result we use a cover of the plane by maximal parallelizable regions and relations between parallelizing homeomorphisms of these regions. We show that for topologically equivalent Brouwer flows there exists a one-to-one correspondence between such covers of the plane.
\end{abstract}

Keywords Brouwer homeomorphism - Brouwer flow · First prolongational limit set · Topological equivalence · Topological conjugacy

Mathematics Subject Classification Primary 39B12; Secondary 54H20, 37E30

\section{Introduction}

In this paper we deal with the problem of topological conjugacy of Brouwer flows. In $[2,6,16]$ one can find necessary and sufficient conditions for such flows to be topologically equivalent. Topologically conjugate flows are topologically equivalent, but not conversely. In [3] it has been constructed an uncountable family of topologically equivalent Brouwer flows such that any two elements of the family are not topological conjugate. Our aim is to give a sufficient and necessary condition for topologically equivalent Brouwer flows to be topologically conjugate.

\section{Communicated by Rosihan M. Ali.}

Zbigniew Leśniak

zlesniak@up.krakow.pl

1 Department of Mathematics, Pedagogical University, Podchorążych 2, 30-084 Kraków, Poland 
After introducing basic notions concerning Brouwer flows, we recall the decomposition theorem for such flows used in the proof of the main result of this paper. We also present properties of the transition maps between parallelizing homeomorphisms and prove an extension result. In Sect. 2 we prove that for topologically equivalent Brouwer flows one can take the same admissible class of finite sequences occurring in the decomposition theorem. Section 3 contains the statement and the proof of the main result of this paper. It gives a sufficient condition for topologically equivalent Brouwer flows to be topologically conjugate. At the end of this paper we prove that this condition is also necessary.

Now we briefly recall the notions that will be used in this paper. A more detailed introduction concerning Brouwer flows can be found in $[15,16,20]$.

By a flow we mean a family $\left\{f^{t}: t \in \mathbb{R}\right\}$ of homeomorphisms of a topological space $X$ onto itself such that

$$
f^{t} \circ f^{s}=f^{t+s}, \quad t, s \in \mathbb{R},
$$

and the function $F: X \times \mathbb{R} \rightarrow X$ given by $F(x, t)=f^{t}(x)$ is continuous. Let us note that any flow $\left\{f^{t}: t \in \mathbb{R}\right\}$ is a group under the operation of composition, where $f^{0}$ is the identity function and the inverse of $f^{t}$ is equal to $f^{-t}$ for $t \in \mathbb{R}$.

Let $\left\{f^{t}: t \in \mathbb{R}\right\}$ and $\left\{g^{t}: t \in \mathbb{R}\right\}$ be flows defined on topological spaces $X$ and $Y$, respectively. Then they are said to be topologically equivalent if there exists a homeomorphism $\Phi: X \rightarrow Y$ mapping trajectories of $\left\{f^{t}: t \in \mathbb{R}\right\}$ onto trajectories of $\left\{g^{t}: t \in \mathbb{R}\right\}$ and preserving the orientations of the trajectories (see [9, p. 32]). We say that the flows are topologically conjugate if there exists a homeomorphism $\Phi: X \rightarrow Y$ such that

$$
\Phi\left(f^{t}(x)\right)=g^{t}(\Phi(x)), \quad x \in X, t \in \mathbb{R} .
$$

For a flow $\left\{f^{t}: t \in \mathbb{R}\right\}$ defined on a Hausdorff space $X$ and a point $p \in X$ one can define the first positive prolongational limit set and the first negative prolongational limit set of $p$ by

$\mathrm{J}_{f}^{+}(p):=\left\{q \in X: \quad\right.$ there exist sequences $\left(p_{n}\right)_{n \in \mathbb{Z}_{+}}$and $\left(t_{n}\right)_{n \in \mathbb{Z}_{+}}$such that $p_{n} \rightarrow p, t_{n} \rightarrow+\infty, f^{t_{n}}\left(p_{n}\right) \rightarrow q$ as $\left.n \rightarrow+\infty\right\}$,

$\mathrm{J}_{f}^{-}(p):=\left\{q \in X: \quad\right.$ there exist sequences $\left(p_{n}\right)_{n \in \mathbb{Z}_{+}}$and $\left(t_{n}\right)_{n \in \mathbb{Z}_{+}}$such that $p_{n} \rightarrow p, t_{n} \rightarrow-\infty, f^{t_{n}}\left(p_{n}\right) \rightarrow q$ as $\left.n \rightarrow+\infty\right\}$

(cf. [4, p. 25]). Moreover, let us put

$$
\mathbf{J}_{f}(p):=\mathbf{J}_{f}^{+}(p) \cup \mathbf{J}_{f}^{-}(p)
$$

for every $p \in X$ and

$$
\mathrm{J}_{f}(H):=\bigcup_{p \in H} \mathrm{~J}_{f}(p)
$$

for all $H \subset X$.

Now let us restrict to a class of flows defined on $\mathbb{R}^{2}$. A flow $\left\{f^{t}: t \in \mathbb{R}\right\}$ will be called a Brouwer flow if it contains a Brouwer homeomorphism, where by a Brouwer 
homeomorphism we mean an orientation-preserving homeomorphism of the plane onto itself which has no fixed points (cf. [5,8]). It is known that if $\left\{f^{t}: t \in \mathbb{R}\right\}$ is a Brouwer flow, then $f^{t}$ is a Brouwer homeomorphism for each $t \in \mathbb{R} \backslash\{0\}$ (see [1]).

For each $p \in \mathbb{R}^{2}$ let us denote by $C_{p}$ the trajectory of $p$, i.e., $C_{p}:=\left\{f^{t}(p): t \in \mathbb{R}\right\}$. Each trajectory of a Brouwer flow is an unbounded closed set and divides the plane into two invariant unbounded simply connected regions (see [1]). Thus, every Brouwer flow is a plane flow with no compact trajectories. Conversely, if a flow $\left\{f^{t}: t \in \mathbb{R}\right\}$ has no fixed points and no periodic trajectories, then for each $t \in \mathbb{R} \backslash\{0\}$ the homeomorphism $f^{t}$ has no fixed points. Thus, $\left\{f^{t}: t \in \mathbb{R}\right\}$ is a Brouwer flow, since every element of a plane flow has to preserve orientation. Hence, every trajectory of such flow is a closed subset of the plane. This result can be also obtained from a generalization of the Poincaré-Bendixson theorem which can be found in [7].

In this paper we study plane flows with trajectories which are unbounded and closed sets. Therefore, any two different trajectories $C_{p_{1}}, C_{p_{2}}$ determine the strip, i.e., the component of $\mathbb{R}^{2} \backslash\left(C_{p_{1}} \cup C_{p_{2}}\right)$ which contains $C_{p_{1}}$ and $C_{p_{2}}$ in its boundary. The strip between $C_{p_{1}}$ and $C_{p_{2}}$ will be denoted by $D_{p_{1} p_{2}}$. It is an invariant simply connected region.

For any distinct trajectories $C_{p_{1}}, C_{p_{2}}, C_{p_{3}}$ of $\left\{f^{t}: t \in \mathbb{R}\right\}$ one of the following two possibilities must be satisfied: Exactly one of the trajectories $C_{p_{1}}, C_{p_{2}}, C_{p_{3}}$ is contained in the strip between the other two, or each of the trajectories $C_{p_{1}}, C_{p_{2}}, C_{p_{3}}$ is contained in the strip between the other two. In the first case if $C_{p_{j}}$ is the trajectory which lies in the strip between $C_{p_{i}}$ and $C_{p_{k}}$ we will write $C_{p_{i}}\left|C_{p_{j}}\right| C_{p_{k}}(i, j, k \in$ $\{1,2,3\}$ and $i, j, k$ are different). In the second case we will write $\left|C_{p_{i}}, C_{p_{j}}, C_{p_{k}}\right|$ (cf. $[10])$.

Let $\left\{f^{t}: t \in \mathbb{R}\right\}$ be a Brouwer flow and $U \subset \mathbb{R}^{2}$ be a simply connected invariant region, i.e., $f^{t}(U)=U$ for $t \in \mathbb{R}$. We say that $U$ is a parallelizable region if there exists a homeomorphism $\varphi_{f, U}$ mapping $U$ onto $\mathbb{R}^{2}$ such that

$$
f^{t}(x)=\varphi_{f, U}^{-1}\left(\varphi_{f, U}(x)+(t, 0)\right), \quad x \in U, t \in \mathbb{R} .
$$

Such a homeomorphism $\varphi_{f, U}$ will be called a parallelizing homeomorphism of the flow $\left\{\left.f^{t}\right|_{U}: t \in \mathbb{R}\right\}$. On account of the Whitney-Bebutov theorem (see [4, p. 52]), for each $p \in \mathbb{R}^{2}$ there exists a parallelizable region $U_{p}$ such that $p \in U_{p}$.

We will consider a cover of the plane by maximal parallelizable regions, i.e., such parallelizable regions which are contained properly in no other parallelizable region. It is known that if $U$ is a maximal parallelizable region, then $\mathrm{J}_{f}(U)=$ bd $U$ (see [19]). As the set of indices of such a cover we take a class of finite sequences of integers described below.

Let $X$ be a nonempty set. Denote by $X^{<\omega}$ the set of all finite sequences of elements of $X$. By a tree on $X$ we mean a subset $T$ of $X^{<\omega}$ which is closed under initial segments, i.e., for all positive integers $m, n$ such that $n>m$, if $\left(x_{1}, \ldots, x_{m}, \ldots, x_{n}\right) \in T$, then $\left(x_{1}, \ldots, x_{m}\right) \in T$.

Let $\alpha=\left(x_{1}, \ldots, x_{n}\right) \in X^{<\omega}$. Then, for any $x \in X$ by $\widehat{\alpha x}$ we denote the sequence $\left(x_{1}, \ldots, x_{n}, x\right)$. If trees $A^{+} \subset \mathbb{Z}_{+}^{<\omega}$ and $A^{-} \subset \mathbb{Z}_{-}^{<\omega}$ satisfy the conditions

(i) $A^{+}$contains the sequence 1 and no other one-element sequence, 


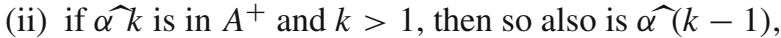

(iii) $A^{-}$contains the sequence -1 and no other one-element sequence,

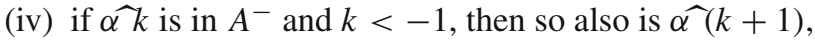

then the set $A:=A^{+} \cup A^{-}$will be said to be admissible class of finite sequences.

Now we can recall the main results describing the structure of Brouwer flows. The first of them says that for any Brouwer flow there exists a cover of the plane consisting of maximal parallelizable regions which can be indexed in a convenient way by an admissible class of finite sequences. The second one describes relations between the parallelizing homeomorphisms of overlapping elements of the cover.

Theorem 1.1 (See [11]) Let $\left\{f^{t}: t \in \mathbb{R}\right\}$ be a Brouwerflow. Then there exist a family of trajectories $\left\{C_{\alpha}: \alpha \in A\right\}$ and a family of maximal parallelizable regions $\left\{U_{\alpha}: \alpha \in A\right\}$, where $A=A^{+} \cup A^{-}$is an admissible class of finite sequences, such that $U_{1}=$ $U_{-1}, C_{1}=C_{-1}$ and

$$
\begin{aligned}
& C_{\alpha} \subset U_{\alpha} \text { for } \alpha \in A \text {, } \\
& \bigcup_{\alpha \in A} U_{\alpha}=\mathbb{R}^{2} \\
& U_{\alpha} \cap U_{\widehat{\alpha i}} \neq \emptyset \text { for } \widehat{\alpha i} \in A \text {, } \\
& C_{\alpha \widehat{i}} \subset \operatorname{bd} U_{\alpha} \text { for } \widehat{\alpha i} \in A \text {, } \\
& \left|C_{\alpha}, C_{\alpha \vec{i}_{1}}, C_{\alpha i_{2}}\right| \text { for } \widehat{\alpha i_{1}}, \widehat{\alpha i_{2}} \in A, \quad i_{1} \neq i_{2} \text {, } \\
& C_{\alpha}\left|C_{\alpha \widehat{i}}\right| C_{\alpha \widehat{\imath} j} \text { for } \widehat{\alpha \imath \hat{l} j} \in A \text {. }
\end{aligned}
$$

Theorem 1.2 (See [11]) Let $\left\{f^{t}: t \in \mathbb{R}\right\}$ be a Brouwer flow. Then there exists a family of the parallelizing homeomorphisms $\left\{\varphi_{\alpha}: \alpha \in A^{+}\right\}$, where $\varphi_{\alpha}: U_{\alpha} \rightarrow \mathbb{R}^{2}, U_{\alpha}$ are those occurring in Theorem 1.1, such that for each $\widehat{\alpha i} \in A^{+}$

$$
\begin{aligned}
\varphi_{\alpha \widehat{i}}\left(U_{\alpha} \cap U_{\alpha \widehat{i}}\right) & =\mathbb{R} \times\left(c_{\widehat{\alpha i}}, 0\right), \\
\varphi_{\alpha}\left(U_{\alpha} \cap U_{\alpha \widehat{i}}\right) & =\mathbb{R} \times\left(c_{\widehat{\alpha i}}^{\alpha}, d_{\widehat{\alpha i}}^{\alpha}\right),
\end{aligned}
$$

where $c_{\widehat{\alpha i}}^{\alpha} \in \mathbb{R} \cup\{-\infty\}, d_{\widehat{\alpha i}}^{\alpha} \in \mathbb{R} \cup\{+\infty\}$ and $c_{\widehat{\alpha i}} \in[-\infty, 0)$ are some constants such that $c_{\widehat{\alpha i}}^{\alpha}<d_{\widehat{\alpha i}}^{\alpha}$ and at least one of the constants $c_{\widehat{\alpha i}}^{\alpha}, d_{\widehat{\alpha i}}^{\alpha}$ is finite. Moreover, there exist a continuous function $\mu_{\widehat{\alpha} \hat{i}}:\left(c_{\widehat{\alpha i}}^{\alpha}, d_{\widehat{\alpha i}}^{\alpha}\right) \rightarrow \mathbb{R}$ and a homeomorphism $v_{\alpha \widehat{i}}:\left(c_{\widehat{\alpha i}}^{\alpha}, d_{\widehat{\alpha i}}^{\alpha}\right) \rightarrow\left(c_{\alpha \widehat{i}}, 0\right)$ such that the homeomorphism

$$
h_{\alpha \widehat{i}}: \mathbb{R} \times\left(c_{\widehat{\alpha i}}^{\alpha}, d_{\widehat{\alpha i}}^{\alpha}\right) \rightarrow \mathbb{R} \times\left(c_{\widehat{\alpha i}}, 0\right)
$$

given by the relation

$$
h_{\alpha \widehat{i}}:=\varphi_{\widehat{\alpha i}} \circ\left(\varphi_{\left.\alpha\right|_{U_{\alpha} \cap U_{\widehat{\alpha i}}}}\right)^{-1}
$$

has the form

$$
h_{\widehat{\alpha i}}(t, s)=\left(\mu_{\alpha \widehat{i}}(s)+t, v_{\widehat{\alpha i}}(s)\right), \quad t \in \mathbb{R}, s \in\left(c_{\widehat{\alpha i}}^{\alpha}, d_{\widehat{\alpha i}}^{\alpha}\right) .
$$


Similarly, there exists a family of the parallelizing homeomorphisms $\left\{\varphi_{\alpha}: \alpha \in A^{-}\right\}$, where $\varphi_{\alpha}: U_{\alpha} \rightarrow \mathbb{R}^{2}, U_{\alpha}$ are those occurring in Theorem $1.1, \varphi_{-1}=\varphi_{1}$, such that for each $\widehat{\alpha i} \in A^{-}$

$$
\begin{gathered}
\varphi_{\alpha \widehat{i}}\left(U_{\alpha} \cap U_{\widehat{\alpha i}}\right)=\mathbb{R} \times\left(0, c_{\alpha \widehat{i}}\right), \\
\varphi_{\alpha}\left(U_{\alpha} \cap U_{\widehat{\alpha i}}\right)=\mathbb{R} \times\left(c_{\alpha \widehat{i}}^{\alpha}, d_{\alpha \widehat{\alpha i}}^{\alpha}\right),
\end{gathered}
$$

where $c_{\widehat{\alpha i}}^{\alpha} \in \mathbb{R} \cup\{-\infty\}, d_{\widehat{\alpha i}}^{\alpha} \in \mathbb{R} \cup\{+\infty\}$ and $c_{\widehat{\alpha i}} \in(0,+\infty)$ are some constants such that $c_{\widehat{\alpha i}}^{\alpha}<d_{\widehat{\alpha i}}^{\alpha}$ and at least one of the constants $c_{\widehat{\alpha i}}^{\alpha}, d_{\widehat{\alpha i}}^{\alpha}$ is finite. Moreover, there exist a continuous function $\mu_{\widehat{\alpha} \hat{i}}:\left(c_{\widehat{\alpha i}}^{\alpha}, d_{\widehat{\alpha} \hat{i}}^{\alpha}\right) \rightarrow \mathbb{R}$ and a homeomorphism $v_{\alpha \widehat{i}}:\left(c_{\widehat{\alpha i}}^{\alpha}, d_{\widehat{\alpha \imath}}^{\alpha}\right) \rightarrow\left(0, c_{\widehat{\alpha i}}\right)$ such that the homeomorphism

$$
h_{\alpha \widehat{i}}: \mathbb{R} \times\left(c_{\widehat{\alpha i}}^{\alpha}, d_{\widehat{\alpha} \widehat{i}}^{\alpha}\right) \rightarrow \mathbb{R} \times\left(0, c_{\alpha \widehat{i}}\right)
$$

given by relation (7) satisfies (8).

The construction of the families occurring in Theorem 1.1 starts from a trajectory denoted by $C_{1}=C_{-1}$ and a maximal parallelizable region $U_{1}=U_{-1}$. Moreover, we denote by $H_{1}$ and $H_{-1}$ the components of $\mathbb{R}^{2} \backslash C_{1}$. Having constructed an index $\alpha \in A$ and the corresponding $C_{\alpha}, U_{\alpha}$ and $H_{\alpha}$ we denote, in case bd $U_{\alpha} \cap H_{\alpha} \neq \emptyset$, by $C_{\alpha \hat{\imath}}$ the trajectories contained in bd $U_{\alpha} \cap H_{\alpha}$ starting from $i=1$ and taking subsequent positive integers $i$ if $\alpha \in A^{+}$and starting from $i=-1$ and taking subsequent negative integers $i$ if $\alpha \in A^{-}$. Then for each trajectory $C_{\alpha \widehat{i}}$ we take a maximal parallelizable region $U_{\alpha \widehat{\imath}}$ containing it and denote by $H_{\alpha \widehat{i}}$ the component of $\mathbb{R}^{2} \backslash C_{\widehat{\alpha i}}$ which does not contain $U_{\alpha}$.

The parallelizing homeomorphisms $\varphi_{\alpha}: U_{\alpha} \rightarrow \mathbb{R}^{2}$ occurring in Theorem 1.2 satisfy the conditions $\varphi_{\alpha}\left(C_{\alpha}\right)=\mathbb{R} \times\{0\}$ and $\varphi_{\alpha}\left(U_{\alpha} \cap H_{\alpha}\right)=\mathbb{R} \times(0,+\infty)$. Each of these homeomorphisms maps trajectories contained in $U_{\alpha}$ onto horizontal straight lines. The parallelizing homeomorphisms will be also called charts.

In the next part of this section we prove that the homeomorphisms $v_{\alpha \widehat{i}}$ occurring in Theorem 1.2 can be extended to one of the endpoints of the interval $\left(c_{\alpha \overleftrightarrow{\alpha}}^{\alpha}, d_{\alpha \overleftrightarrow{\imath}}^{\alpha}\right)$. This result will be used in the main part of this paper.

The homeomorphisms $v_{\alpha \widehat{i}}$ can be either increasing or decreasing. The extension of the homeomorphism $v_{\alpha \hat{i}}$ will contain the number 0 in its image. If such an extension exists, then $v_{\alpha \widehat{i}}\left(c_{\widehat{\alpha i}}^{\alpha}\right)=0$ in the case where $v_{\alpha \widehat{i}}$ is decreasing, and $v_{\alpha \widehat{i}}\left(d_{\widehat{\alpha i}}^{\alpha}\right)=0$ in the case where $v_{\alpha \hat{i}}$ is increasing. The necessary condition for the existence of such an extension is the correspondence by $\varphi_{\alpha}$ between $v_{\alpha \hat{\alpha} i}^{-1}(0)$ and a trajectory contained in $U_{\alpha}$. In the chart $\varphi_{\alpha \widehat{i}}$ the number 0 corresponds to $C_{\alpha \widehat{i}}$, since the second coordinate in $\varphi_{\alpha \widehat{i}}$ of every point of $C_{\alpha \widehat{i}}$ is equal to 0 , i.e., $\varphi_{\widehat{\alpha} \vec{i}}\left(C_{\alpha \widehat{i}}\right)=\mathbb{R} \times\{0\}$.

To find a trajectory which corresponds to $v_{\alpha \hat{i}}^{-1}(0)$ we will use properties of the first prolongational limit set of boundary trajectories of a parallelizable region.

Proposition 1.3 (See [12]) Let $U$ be a parallelizable region of $\left\{f^{t}: t \in \mathbb{R}\right\}$. Let $p \in \operatorname{bd} U$ and $q_{1}, q_{2} \in U$. Assume that $q_{1}, q_{2} \in \mathrm{J}_{f}(p)$. Then $C_{q_{1}}=C_{q_{2}}$.

Using the above proposition one can obtain the following corollary. 
Corollary 1.4 Let $U$ be a maximal parallelizable region of $\left\{f^{t}: t \in \mathbb{R}\right\}$. Let $p \in$ bd $U$. Then the set $\mathrm{J}_{f}(p) \cap U$ consists of exactly one trajectory.

Proof Since $U$ is a maximal parallelizable region of the flow $\left\{f^{t}: t \in \mathbb{R}\right\}$, we have bd $U=\mathrm{J}_{f}(U)$. Hence, $\mathrm{J}_{f}(p) \cap U \neq \emptyset$. By Proposition 1.3 each element of the set $\mathrm{J}_{f}(p) \cap U$ belongs to the same trajectory.

For each $\widehat{\alpha i} \in A$ denote by $C_{\widehat{\alpha i}}^{\alpha}$ the unique trajectory contained in $U_{\alpha} \cap \mathrm{J}\left(C_{\alpha \vec{\alpha}}\right)$ occurring in Corollary 1.4. We will show that the trajectory $C_{\widehat{\alpha i}}^{\alpha}$ corresponds either to $c_{\widehat{\alpha i}}^{\alpha}$ or to $d_{\widehat{\alpha i}}^{\alpha}$. This depends on how $C_{\widehat{\alpha i}}^{\alpha}$ is situated in relation to $C_{\alpha}$ and $C_{\alpha \widehat{i}}$. The following result shows that the relation between the trajectories $C_{\widehat{\alpha i}}^{\alpha}, C_{\alpha}$ and $C_{\alpha \hat{i}}$ determines the kind of monotonicity of $v_{\alpha \widehat{i}}$.

Proposition 1.5 (See [11]) If $C_{\alpha}\left|C_{\widehat{\alpha i}}^{\alpha}\right| C_{\alpha \widehat{i}}$ or $C_{\alpha}=C_{\widehat{\alpha i}}^{\alpha}$, then the homeomorphism $v_{\alpha \hat{i}}$ is decreasing and $c_{\widehat{\alpha i}}^{\alpha}>0$ or $c_{\widehat{\alpha i}}^{\alpha}=0$, respectively. However, in case $\left|C_{\alpha}, C_{\widehat{\alpha i}}^{\alpha}, C_{\alpha \widehat{i}}\right|$, the homeomorphism $v_{\alpha \widehat{i}}$ is increasing and $d_{\widehat{\alpha i}}^{\alpha}>0$.

Now we can proceed to the extension result mentioned above.

Proposition 1.6 If $C_{\alpha}\left|C_{\alpha \widehat{i}}^{\alpha}\right| C_{\alpha \widehat{i}}$ or $C_{\alpha}=C_{\widehat{\alpha i}}^{\alpha}$, then $\varphi_{\alpha}\left(C_{\alpha \widehat{\alpha i}}^{\alpha}\right)=\mathbb{R} \times\left\{c_{\widehat{\alpha i}}^{\alpha}\right\}$ and $v_{\alpha \widehat{i}}$ : $\left(c_{\widehat{\alpha i}}^{\alpha}, d_{\widehat{\alpha \imath}}^{\alpha}\right) \rightarrow\left(c_{\widehat{\alpha i}}, 0\right)$ can be extended to a homeomorphism defined on $\left[c_{\widehat{\alpha i}}^{\alpha}, d_{\widehat{\alpha i}}^{\alpha}\right)$

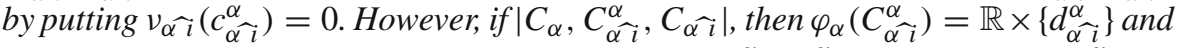
$v_{\alpha \widehat{i}}$ can be extended to a homeomorphism defined on $\left(c_{\widehat{\alpha i}}^{\alpha}, d_{\widehat{\alpha i}}^{\alpha}\right]$ by putting $v_{\alpha \widehat{i}}\left(d_{\widehat{\alpha i}}^{\alpha}\right)=$ 0 .

Proof By the definition of $v_{\alpha \widehat{i}}$ we have $\varphi_{\widehat{\alpha i}}^{-1}\left(\mathbb{R} \times\left\{v_{\widehat{\alpha i}}(s)\right\}\right)=\varphi_{\alpha}^{-1}(\mathbb{R} \times\{s\})$ for $s \in\left(c_{\widehat{\alpha i}}^{\alpha}, d_{\widehat{\alpha i}}^{\alpha}\right)$, i.e., $v_{\alpha \widehat{i}}(s)$ and $s$ correspond to the same trajectory contained in $U_{\alpha} \cap U_{\alpha \widehat{i}}$. First, let us consider the case where $C_{\alpha}\left|C_{\widehat{\alpha i}}^{\alpha}\right| C_{\alpha \widehat{i} i}$ or $C_{\alpha}=C_{\widehat{\alpha i}}^{\alpha}$. Then $v_{\alpha \widehat{i}}$ is a decreasing homeomorphism. Hence, for any sequence $\left(s_{n}\right)_{n \in \mathbb{Z}_{+}}$such that $s_{n} \in$ $\left(c_{\alpha \widehat{\imath}}^{\alpha}, d_{\widehat{\alpha i}}^{\alpha}\right)$ for $n \in \mathbb{Z}_{+}$, we have $\lim _{n \rightarrow \infty} s_{n}=c_{\widehat{\alpha i}}^{\alpha}$ if and only if $\lim _{n \rightarrow \infty} v_{\alpha \widehat{i}}\left(s_{n}\right)=0$, since $C_{\alpha \widehat{i}}^{\alpha} \subset \mathrm{J}_{f}\left(C_{\alpha \widehat{i}}\right)$.

To prove that $\varphi_{\alpha}^{-1}\left(\mathbb{R} \times\left\{c_{\alpha \widehat{l}}^{\alpha}\right\}\right)=C_{\widehat{\alpha i}}^{\alpha}$, we take a sequence $\left(p_{n}\right)_{n \in \mathbb{Z}_{+}}$of points from $U_{\alpha} \cap U_{\widehat{\alpha} \hat{i}}$ which tends to a $p \in C_{\widehat{\alpha i}}$. Then there exists the corresponding sequence $\left(s_{n}\right)_{n \in \mathbb{Z}_{+}}$such that $s_{n} \in\left(c_{\widehat{\alpha i}}^{\alpha}, d_{\widetilde{\alpha i}}^{\alpha}\right), p_{n} \in \varphi_{\alpha \widehat{\alpha}}^{-1}\left(\mathbb{R} \times\left\{v_{\alpha \widehat{i}}\left(s_{n}\right)\right\}\right)$ and $\lim _{n \rightarrow \infty} v_{\alpha \widehat{i}}\left(s_{n}\right)=$ 0 . Since $C_{\widehat{\alpha i}}^{\alpha} \subset \mathrm{J}_{f}\left(C_{\widehat{\alpha i}}\right)$, there exists a sequence $\left(t_{n}\right)_{n \in \mathbb{Z}_{+}}$such that $f^{t_{n}}\left(p_{n}\right) \rightarrow q$ as $n \rightarrow+\infty$ for some $q \in C_{\widehat{\alpha i}}^{\alpha}$. Moreover, $f^{t_{n}}\left(p_{n}\right) \in \varphi_{\widehat{\alpha i}}^{-1}\left(\mathbb{R} \times\left\{v_{\alpha \widehat{i}}\left(s_{n}\right)\right\}\right)=$ $\varphi_{\alpha}^{-1}\left(\mathbb{R} \times\left\{s_{n}\right\}\right)$. Thus, $C_{\widehat{\alpha i}}^{\alpha}$ has to correspond to $c_{\widehat{\alpha i}}^{\alpha}$ by $\varphi_{\alpha}$, since $\lim _{n \rightarrow \infty} s_{n}=c_{\widehat{\alpha i}}^{\alpha}$ and the sequence $\left(f^{t_{n}}\left(p_{n}\right)\right)_{n \in \mathbb{Z}_{+}}$tends to a point of $C_{\alpha \widehat{\alpha}}^{\alpha}$.

The case where $\left|C_{\alpha}, C_{\widehat{\alpha i}}^{\alpha}, C_{\alpha \widehat{i}}\right|$ is similar. Then $v_{\alpha \hat{i}}$ is an increasing homeomorphism. Replacing in the above consideration $c_{\widehat{\alpha i}}^{\alpha}$ by $d_{\widehat{\alpha i}}^{\alpha}$, we obtain that $\varphi_{\alpha}^{-1}\left(\mathbb{R} \times\left\{d_{\alpha \hat{\alpha}}^{\alpha}\right\}\right)=C_{\widehat{\alpha i}}^{\alpha}$.

The continuous functions $\mu_{\alpha \hat{i}}$ occurring in Theorem 1.2 describe the time needed for the flow $\left\{f^{t}: t \in \mathbb{R}\right\}$ to move from the point with coordinates $\left(0, v_{\alpha \widehat{i}}(s)\right)$ in the chart $\varphi_{\alpha \widehat{i}}$ until it reaches the point with coordinates $(0, s)$ in the chart $\varphi_{\alpha}$. In other words, $\mu_{\widehat{\alpha i}}$ describe the time needed for the flow to move from a point from the section $K_{\varphi_{\alpha \widehat{i}}}$ in $U_{\alpha \widehat{\alpha i}}$ to a point from the section $K_{\varphi_{\alpha}}$ in $U_{\alpha}$, where $K_{\varphi_{\alpha \widehat{i}}}:=\varphi_{\widehat{\alpha} i}^{-1}(\{0\} \times \mathbb{R})$ and $K_{\varphi_{\alpha}}:=\varphi_{\alpha}^{-1}(\{0\} \times \mathbb{R})$. 
The following result describes the limits of sequences $\left(\mu_{\alpha \hat{i}}\left(s_{n}\right)\right)_{n \in \mathbb{Z}_{+}}$analogous to sequences $\left(v_{\widehat{\alpha} \hat{i}}\left(s_{n}\right)\right)_{n \in \mathbb{Z}_{+}}$considered in the proof of Proposition 1.6.

Proposition 1.7 (See [13]) The functions $\mu_{\alpha \widehat{i}}$ occurring in Theorem 1.2 satisfy the condition

$$
\lim _{s \rightarrow c_{\alpha \widehat{i}}^{\alpha}} \mu_{\widehat{\alpha i}}(s)=\left\{\begin{array}{lll}
-\infty & \text { if } & C_{\widehat{\alpha i}} \subset \mathrm{J}_{f}^{+}\left(C_{\widehat{\alpha i}}^{\alpha}\right), \\
+\infty & \text { if } & C_{\widehat{\alpha i}} \subset \mathrm{J}_{f}^{-}\left(C_{\widehat{\alpha i}}\right)
\end{array}\right.
$$

in the case where $C_{\alpha}\left|C_{\widehat{\alpha i}}^{\alpha}\right| C_{\alpha \hat{\alpha}}$ or $C_{\alpha}=C_{\widehat{\alpha i}}^{\alpha}$, or the condition

$$
\lim _{s \rightarrow d_{\widehat{\alpha i}}^{\alpha}} \mu_{\widehat{\alpha i}}(s)=\left\{\begin{array}{lll}
-\infty & \text { if } & C_{\widehat{\alpha i}} \subset \mathrm{J}_{f}^{+}\left(C_{\widehat{\alpha i}}^{\alpha}\right), \\
+\infty & \text { if } & C_{\widehat{\alpha i}} \subset \mathrm{J}_{f}^{-}\left(C_{\widehat{\alpha i}}\right)
\end{array}\right.
$$

in the case where $\left|C_{\alpha}, C_{\widehat{\alpha i}}^{\alpha}, C_{\alpha \widehat{i}}\right|$.

Proposition 1.7 describes the property that the trajectories $C_{\alpha \widehat{i}}$ and $C_{\widehat{\alpha i}}^{\alpha}$ cannot be contained in the same parallelizable region, whereas Proposition 1.6 corresponds to the fact that the trajectories $C_{\alpha \widehat{i}}$ and $C_{\widehat{\alpha i}}^{\alpha}$ have no disjoint invariant neighborhoods.

\section{Topological Equivalence of Brouwer Flows}

In this part we present results concerning the topological equivalence of Brouwer flows. In particular, we prove a result about relations between covers of the plane by families of maximal parallelizable regions for topologically equivalent Brouwer flows.

Topologically equivalent Brouwer flows considered in this section will be denoted by $\left\{f^{t}: t \in \mathbb{R}\right\}$ and $\left\{g^{t}: t \in \mathbb{R}\right\}$. Therefore, to distinguish between corresponding elements of the families occurring in Theorem 1.1 we will add the letters $f$ and $g$ to the subscripts. For instance, the maximal parallelizable regions will be denoted by $U_{f, \alpha}$ and $U_{g, \alpha}$, respectively.

We start from a property of the topological equivalence of Brouwer flows concerning maximal parallelizable regions.

Proposition 2.1 Let $\left\{f^{t}: t \in \mathbb{R}\right\}$ and $\left\{g^{t}: t \in \mathbb{R}\right\}$ be topologically equivalent Brouwer flows and $\Phi: \mathbb{R}^{2} \rightarrow \mathbb{R}^{2}$ be a homeomorphism which realizes the equivalence. Then if $U$ is a maximal parallelizable region of the flow $\left\{f^{t}: t \in \mathbb{R}\right\}$, then $\Phi(U)$ is a maximal parallelizable region of the flow $\left\{g^{t}: t \in \mathbb{R}\right\}$.

Proof Let $U$ be a maximal parallelizable region of the flow $\left\{f^{t}: t \in \mathbb{R}\right\}$. Then there exists a section $K$ in $U$. Since $\Phi$ is a homeomorphism, the set $\Phi(K)$ is a section in $\Phi(U)$. Hence, $\Phi(U)$ is a parallelizable region of the flow $\left\{g^{t}: t \in \mathbb{R}\right\}$. Suppose that $\Phi(U)$ is not a maximal parallelizable region of the flow $\left\{g^{t}: t \in \mathbb{R}\right\}$. Then there exists a parallelizable region $U^{\prime}$ extending $\Phi(U)$. Using the same reasoning as above, we obtain that for $\Phi^{-1}\left(U^{\prime}\right)$ is a parallelizable region of the flow $\left\{f^{t}: t \in \mathbb{R}\right\}$, since $\Phi^{-1}$ realizes the topological equivalence of $\left\{g^{t}: t \in \mathbb{R}\right\}$ and $\left\{f^{t}: t \in \mathbb{R}\right\}$. Moreover, we have $U \subset \Phi^{-1}\left(U^{\prime}\right)$ and $U \neq \Phi^{-1}\left(U^{\prime}\right)$, which is a contradiction. 
In [16] it has been proved that a homeomorphism which realizes the topological equivalence of Brouwer flows preserves the first prolongational limit set. Namely, we have the following result.

Theorem 2.2 (See [16]) Let $\left\{f^{t}: t \in \mathbb{R}\right\}$ and $\left\{g^{t}: t \in \mathbb{R}\right\}$ be topologically equivalent Brouwer flows and $\Phi: \mathbb{R}^{2} \rightarrow \mathbb{R}^{2}$ be a homeomorphism which realizes the equivalence. Then $\Phi\left(\mathbf{J}_{f}\left(\mathbb{R}^{2}\right)\right)=\mathrm{J}_{g}\left(\mathbb{R}^{2}\right)$, where $\mathbf{J}_{f}\left(\mathbb{R}^{2}\right)$ and $\mathbf{J}_{g}\left(\mathbb{R}^{2}\right)$ denote the first prolongational limit set of $\left\{f^{t}: t \in \mathbb{R}\right\}$ and $\left\{g^{t}: t \in \mathbb{R}\right\}$, respectively.

In the main step of the proof of this result it has been showed that if $q \in \mathrm{J}_{f}(p)$, then $\Phi(q) \in \mathrm{J}_{g}(\Phi(p))$, where $\Phi: \mathbb{R}^{2} \rightarrow \mathbb{R}^{2}$ is a homeomorphism which realizes the topological equivalence of $\left\{f^{t}: t \in \mathbb{R}\right\}$ and $\left\{g^{t}: t \in \mathbb{R}\right\}$. To prove this fact some properties of the first prolongational limit set given in [14] have been used. Therefore, we can state the following result.

Proposition 2.3 Let $\left\{f^{t}: t \in \mathbb{R}\right\}$ and $\left\{g^{t}: t \in \mathbb{R}\right\}$ be topologically equivalent Brouwer flows and $\Phi: \mathbb{R}^{2} \rightarrow \mathbb{R}^{2}$ be a homeomorphism which realizes the equivalence. Then for all $p, q \in \mathbb{R}^{2}$ if $q \in \mathrm{J}_{f}(p)$, then $\Phi(q) \in \mathrm{J}_{g}(\Phi(p))$.

For a given Brouwer flow $\left\{f^{t}: t \in \mathbb{R}\right\}$ let us consider families $\left\{C_{f, \alpha}: \alpha \in A\right\}$ and $\left\{U_{f, \alpha}: \alpha \in A\right\}$ of trajectories and maximal parallelizable regions, respectively, occurring in Theorem 1.1. We will find corresponding trajectories $C_{g, \alpha}$ and maximal parallelizable regions $U_{g, \alpha}$ for any Brouwer flow $\left\{g^{t}: t \in \mathbb{R}\right\}$ topologically equivalent to $\left\{f^{t}: t \in \mathbb{R}\right\}$. The admissible class of finite sequences occurring in Theorem 1.1 is not unique for a given flow, so we can usually choose a convenient $A$ when solving a problem topological conjugacy.

By Corollary 1.4, for each $\widehat{\alpha i} \in A$ there exists a uniquely determined trajectory $C_{f, \widehat{\alpha i}}^{\alpha}$ contained in $\mathrm{J}_{f}\left(C_{f, \widehat{\alpha i}}\right) \cap U_{f, \alpha}$. It plays an important role in Propositions 1.6 and 1.7 describing the properties of $v_{\alpha \hat{i}}$ and $\mu_{\alpha \widehat{i}}$. Similarly, for each $\widehat{\alpha \lambda} \in A$ there exists a unique trajectory $C_{g, \widehat{\alpha} \widehat{\alpha}}^{\alpha}$ contained in $\mathrm{J}_{g}\left(C_{g, \widehat{\alpha i}}\right) \cap U_{g, \alpha}$. The main part of the proof of the next theorem is to show that any homeomorphism which realizes the topological equivalence maps $C_{f, \alpha \widehat{\alpha}}^{\alpha}$ onto $C_{g, \alpha \widehat{i}}^{\alpha}$.

Theorem 2.4 Let $\left\{f^{t}: t \in \mathbb{R}\right\}$ and $\left\{g^{t}: t \in \mathbb{R}\right\}$ be topologically equivalent Brouwer flows and $\Phi: \mathbb{R}^{2} \rightarrow \mathbb{R}^{2}$ be a homeomorphism which realizes the equivalence. Let $\left\{C_{f, \alpha}: \alpha \in A\right\}$ be a family of trajectories and $\left\{U_{f, \alpha}: \alpha \in A\right\}$ a family of maximal parallelizable regions of $\left\{f^{t}: t \in \mathbb{R}\right\}$, where $A=A^{+} \cup A^{-}$is an admissible class of finite sequences, such that $U_{f, 1}=U_{f,-1}, C_{f, 1}=C_{f,-1}$ and conditions (1)-(6) are satisfied. Define $C_{g, \alpha}:=\Phi\left(C_{f, \alpha}\right)$ and $U_{g, \alpha}:=\Phi\left(U_{f, \alpha}\right)$ for $\alpha \in$ A. Then $\left\{C_{g, \alpha}: \alpha \in\right.$ $A\}$ is a family of trajectories and $\left\{U_{g, \alpha}: \alpha \in A\right\}$ is a family of maximal parallelizable regions of $\left\{g^{t}: t \in \mathbb{R}\right\}$ such that conditions (1)-(6) hold. Moreover, we have

$$
\Phi\left(C_{f, \widehat{\alpha i}}^{\alpha}\right)=C_{g, \widehat{\alpha i}}^{\alpha}, \quad \widehat{\alpha i} \in A,
$$

where $C_{f, \widehat{\alpha i}}^{\alpha}$ and $C_{g, \widehat{\alpha} \widehat{\alpha}}^{\alpha}$ are the unique trajectories contained in $\mathrm{J}_{f}\left(C_{f, \widehat{\alpha i}}\right) \cap U_{f, \alpha}$ and $\mathrm{J}_{g}\left(C_{g, \alpha i}\right) \cap U_{g, \alpha}$, respectively. 
Proof By Proposition 2.1, the set $U_{g, \alpha}$ is a maximal parallelizable region of $\left\{g^{t}: t \in \mathbb{R}\right\}$ for each $\alpha \in A$.

From the assumption that $\Phi$ realizes the topological equivalence we obtain that $C_{g, \alpha}$ is a trajectory of the flow $\left\{g^{t}: t \in \mathbb{R}\right\}$. Since $\Phi$ is a homeomorphism, conditions (1)-(6) are satisfied. In particular, conditions (5) and (6) hold, since every homeomorphism preserves the considered ternary relations.

Let us fix an $\alpha \in A$. Put $I_{\alpha}:=\{i \in \mathbb{Z}: \widehat{\alpha i} \in A\}$, and take any $i \in I_{\alpha}$. By Proposition 2.3 the trajectory $\Phi\left(C_{f, \widehat{\alpha i}}^{\alpha}\right)$ is contained in $\mathrm{J}_{g}\left(C_{g, \widehat{\alpha i}}\right)$. Moreover, $\Phi\left(C_{f, \widehat{\alpha i}}^{\alpha}\right) \subset U_{g, \alpha}$, since $C_{f, \alpha \widehat{i}}^{\alpha} \subset U_{f, \alpha}$. Thus, on account of Corollary 1.4, we have $\Phi\left(C_{f, \widehat{\alpha i}}^{\alpha}\right)=C_{g, \widehat{\alpha i}}^{\alpha}$.

For Brouwer flows $\left\{f^{t}: t \in \mathbb{R}\right\}$ and $\left\{g^{t}: t \in \mathbb{R}\right\}$ let us consider the homeomorphisms $v_{f, \widehat{\alpha} i}$ and $v_{g, \widehat{\alpha} \hat{i}}$, respectively, occurring in Theorem 1.2. By Proposition 1.5 the monotonicity of these homeomorphisms depends on the trajectories $C_{f, \alpha}, C_{f, \widehat{\alpha i}}^{\alpha}, C_{f, \widehat{\alpha i}}$ and $C_{g, \alpha}, C_{g, \widehat{\alpha i}}^{\alpha}, C_{\left\{g^{t}\right\}, \widehat{\alpha i}}$, respectively. Therefore, from the above theorem we obtain the following result.

Corollary 2.5 Let $\left\{f^{t}: t \in \mathbb{R}\right\}$ and $\left\{g^{t}: t \in \mathbb{R}\right\}$ be topologically equivalent Brouwer flows. Then for each $\widehat{\alpha i} \in A$ the homeomorphisms $v_{f, \widehat{\alpha i}}$ and $v_{g, \widehat{\alpha i}}$ occurring in Theorem 1.2 are either both increasing or both decreasing.

Proof Let $\Phi: \mathbb{R}^{2} \rightarrow \mathbb{R}^{2}$ be the homeomorphism occurring in Theorem 2.4. Then $\Phi\left(C_{f, \alpha}\right)=C_{g, \alpha}, \Phi\left(C_{f, \widehat{\alpha i}}^{\alpha}\right)=C_{g, \widehat{\alpha i}}^{\alpha}, \Phi\left(C_{f, \widehat{\alpha i}}\right)=C_{\left\{g^{t}\right\}, \widehat{\alpha i}}$. Thus, by Proposition 1.5 the homeomorphisms $v_{f, \widehat{\alpha i}}$ and $v_{g, \alpha \hat{i}}$ have the same kind of monotonicity, since $\Phi$ preserves the considered ternary relations.

Let us remark that Brouwer flows having the same admissible class of finite sequences $A$ (in the decomposition described in Theorem 2.4) may not be topologically equivalent. Consider the case $A=\{-1,1,11,12\}$ and $C_{f, 11}^{1}=C_{f, 12}^{1}=$ $C_{f, 1}, C_{g, 11}^{1}=C_{g, 1}, C_{g, 12}^{1} \neq C_{g, 1}$. Then $\mathrm{J}_{f}\left(\mathbb{R}^{2}\right)=C_{f, 1} \cup C_{f, 11} \cup C_{f, 12}$ and $\mathrm{J}_{g}\left(\mathbb{R}^{2}\right)=C_{g, 1} \cup C_{g, 11} \cup C_{g, 12} \cup C_{g, 12}^{1}$. Thus, by Theorem 2.2, the flows $\left\{f^{t}: t \in \mathbb{R}\right\}$ and $\left\{g^{t}: t \in \mathbb{R}\right\}$ are not topologically equivalent.

\section{Topological Conjugacy of Brouwer Flows}

In this section we deal with the problem of topological conjugacy of Brouwer flows. We prove a sufficient condition for Brouwer flows to be topologically conjugate. First let us recall the known result concerning the problem of topological conjugacy of flows defined on the plane.

Consider a subclass of the family of Brouwer flows which consists of all flows for which the first prolongational limit set contains exactly two trajectories. Such flows are called Reeb flows, and each element of this flow except the identity is said to be a Reeb homeomorphism. Let $\left\{f^{t}: t \in \mathbb{R}\right\}$ be a Reeb flow. We take $A=\{-1,1,11\}$ as the admissible class of finite sequences occurring in Theorem 2.4. Then we can have $\mathrm{J}_{f}\left(\mathbb{R}^{2}\right)=C_{f, 1} \cup C_{f, 11}, C_{f,-1}=C_{f, 1}$ and $C_{f, 11}^{1}=C_{f, 11}^{1}$. The flow has two maximal parallelizable regions $U_{f, 1}=U_{f,-1}$ and $U_{f, 11}$. 
Le Roux [17] has given a classification of conjugacy classes of such flows (see also [18]). The result can be stated in the following form.

Theorem 3.1 (See [17]) Let $\left\{f^{t}: t \in \mathbb{R}\right\}$ and $\left\{g^{t}: t \in \mathbb{R}\right\}$ be Reeb flows which have the same trajectories. Then they are topologically conjugate if and only if there exists a homeomorphism $\beta:[0,+\infty) \rightarrow[0,+\infty)$ and a continuous function $\gamma:[0,+\infty) \rightarrow$ $\mathbb{R}$ such that

$$
\mu_{f, 11}=\mu_{g, 11} \circ \beta+\gamma
$$

where $\mu_{f, 11}, \mu_{g, 11}$ are continuous mappings occurring in Theorem 1.2.

In [13] it has been given a sufficient condition for topological conjugacy of Brouwer flows for which the admissible class of finite sequences $A$ contains only sequences with elements equal to 1 or -1 . Such flows have been called generalized Reeb flows. Now we consider the problem of topological conjugacy for any Brouwer flows.

Let $\left\{f^{t}: t \in \mathbb{R}\right\}$ and $\left\{g^{t}: t \in \mathbb{R}\right\}$ be topologically equivalent Brouwer flows and $\Phi: \mathbb{R}^{2} \rightarrow \mathbb{R}^{2}$ be a homeomorphism which realizes the equivalence. Let $\left\{C_{f, \alpha}: \alpha \in A\right\}$ be a family of trajectories and $\left\{U_{f, \alpha}: \alpha \in A\right\}$ a family of maximal parallelizable regions of $\left\{f^{t}: t \in \mathbb{R}\right\}$ occurring in Theorem 1.1, where $A$ is an admissible class of finite sequences. Let $\left\{\varphi_{f, \alpha}: \alpha \in A\right\}$, where $\varphi_{f, \alpha}: U_{f, \alpha} \rightarrow \mathbb{R}^{2}$, be a family of parallelizing homeomorphisms occurring in Theorem 1.2. Define $C_{g, \alpha}:=\Phi\left(C_{f, \alpha}\right)$ and $U_{g, \alpha}:=$ $\Phi\left(U_{f, \alpha}\right)$ for $\alpha \in A$. Then, by Theorem $2.4,\left\{C_{g, \alpha}: \alpha \in A\right\}$ is a family of trajectories and $\left\{U_{g, \alpha}: \alpha \in A\right\}$ is a family of maximal parallelizable regions of $\left\{g^{t}: t \in \mathbb{R}\right\}$ satisfying the assertion of Theorem 1.1. Let $\left\{\varphi_{g, \alpha}: \alpha \in A\right\}$, where $\varphi_{g, \alpha}: U_{g, \alpha} \rightarrow \mathbb{R}^{2}$, be a family of parallelizing homeomorphisms described in Theorem 1.2.

Let us fix $\widehat{\alpha i} \in A^{+}$and put

$$
b_{f, \widehat{\alpha i}}^{\alpha}:= \begin{cases}c_{f, \widehat{\alpha i}}^{\alpha} & \text { if } C_{f, \alpha}\left|C_{f, \widehat{\alpha i}}^{\alpha}\right| C_{f, \widehat{\alpha i}} \text { or } C_{f, \alpha}=C_{f, \widehat{\alpha i}}^{\alpha}, \\ d_{f, \widehat{\alpha i}}^{\alpha} & \text { if }\left|C_{f, \alpha}, C_{f, \widehat{\alpha i}}^{\alpha}, C_{f, \widehat{\alpha i}}\right|,\end{cases}
$$

where $c_{f, \widehat{\alpha i}}^{\alpha}, d_{f, \widehat{\alpha i}}^{\alpha}$ are those occurring in Theorem 1.2. Then

$$
\varphi_{f, \alpha}\left(C_{f, \widehat{\alpha i}}^{\alpha}\right)=\mathbb{R} \times\left\{b_{f, \alpha \widehat{\imath}}^{\alpha}\right\} .
$$

Similarly, let

$$
b_{g, \widehat{\alpha i}}^{\alpha}:= \begin{cases}c_{g, \widehat{\alpha i}}^{\alpha} & \text { if } C_{g, \alpha}\left|C_{g, \widehat{\alpha i}}^{\alpha}\right| C_{g, \widehat{\alpha i}} \text { or } C_{g, \alpha}=C_{g, \widehat{\alpha i}}^{\alpha}, \\ d_{g, \widehat{\alpha i}}^{\alpha} & \text { if }\left|C_{g, \alpha}, C_{g, \widehat{\alpha i}}^{\alpha}, C_{g, \widehat{\alpha i}}\right| .\end{cases}
$$

Then,

$$
\varphi_{g, \alpha}\left(C_{g, \widehat{\alpha i}}^{\alpha}\right)=\mathbb{R} \times\left\{b_{g, \widehat{\alpha i}}^{\alpha}\right\} .
$$

Hence,

$$
\left(\varphi_{g, \alpha} \circ \Phi \circ \varphi_{f, \alpha}^{-1}\right)\left(\mathbb{R} \times\left\{b_{f, \alpha \widehat{\alpha}}^{\alpha}\right\}\right)=\mathbb{R} \times\left\{b_{g, \alpha \widehat{i}}^{\alpha}\right\}
$$


since, by Theorem $2.4, \Phi\left(C_{f, \alpha \widehat{\alpha i}}^{\alpha}\right)=C_{g, \alpha \widehat{\alpha}}^{\alpha}$. Thus, for the topologically equivalent flows $\left\{f^{t}: t \in \mathbb{R}\right\}$ and $\left\{g^{t}: t \in \mathbb{R}\right\}$ we have

$$
b_{f, \widehat{\alpha} \hat{i}}^{\alpha}=c_{f, \widehat{\alpha i}}^{\alpha} \quad \text { iff } \quad b_{g, \widehat{\alpha} \hat{i}}^{\alpha}=c_{g, \widehat{\alpha i}}^{\alpha},
$$

since every homeomorphism preserves the considered ternary relations. Moreover, from Proposition 1.5 we get that $b_{f, \widehat{\alpha i}}^{\alpha} \geq 0$ and $b_{g, \alpha \widehat{i}}^{\alpha} \geq 0$ for all $\widehat{\alpha i} \in A^{+}$.

In a similar way we can define $b_{f, \alpha \hat{i}}^{\alpha}$ and $b_{g, \alpha \hat{i}}^{\alpha}$ for $\widehat{\alpha i} \in A^{-}$. Namely, if we put

$$
b_{f, \widehat{\alpha i}}^{\alpha}:=\left\{\begin{array}{lll}
d_{f, \widehat{\alpha i}}^{\alpha} & \text { if } & C_{f, \alpha}\left|C_{f, \widehat{\alpha i}}^{\alpha}\right| C_{f, \widehat{\alpha i}} \text { or } C_{f, \alpha}=C_{f, \widehat{\alpha i}}^{\alpha,}, \\
c_{f, \widehat{\alpha i}}^{\alpha} & \text { if } \quad\left|C_{f, \alpha}, C_{f, \widehat{\alpha i}}^{\alpha}, C_{f, \widehat{\alpha i}}\right|,
\end{array}\right.
$$

and

$$
b_{g, \alpha i}^{\alpha}:=\left\{\begin{array}{lll}
d_{g, \widehat{\alpha i}}^{\alpha} & \text { if } & C_{g, \alpha}\left|C_{g, \widehat{\alpha i}}^{\alpha}\right| C_{g, \widehat{\alpha i}} \text { or } C_{g, \alpha}=C_{g, \widehat{\alpha i}}^{\alpha}, \\
c_{g, \alpha \hat{i}}^{\alpha} & \text { if } \quad\left|C_{g, \alpha}, C_{g, \widehat{\alpha i}}^{\alpha}, C_{g, \widehat{\alpha i}}\right|,
\end{array}\right.
$$

then for the topologically equivalent flows $\left\{f^{t}: t \in \mathbb{R}\right\}$ and $\left\{g^{t}: t \in \mathbb{R}\right\}$ condition (12) also holds for all $\widehat{\alpha i} \in A^{-}$. Moreover, $b_{f, \widehat{\alpha i}}^{\alpha} \leq 0$ and $b_{g, \alpha \widehat{i}}^{\alpha} \leq 0$ for all $\widehat{\alpha i} \in A^{-}$.

Now we can proceed to the proof of a sufficient condition for the topological conjugacy of Brouwer flows. To obtain this result we use the fact that for any Brouwer flow one can find a cover of the plane by maximal parallelizable regions. The trajectories of these regions are mapped onto horizontal straight lines by charts of the cover. After such a linearization we considered transition maps $h_{\alpha \widehat{i}}$ for overlapping regions of this cover. A homeomorphism which conjugates the flows is constructed by induction. The crucial step is to extend the homeomorphism from the regions of the cover onto their boundary trajectories.

Theorem 3.2 Let $\left\{f^{t}: t \in \mathbb{R}\right\}$ and $\left\{g^{t}: t \in \mathbb{R}\right\}$ be topologically equivalent Brouwer flows. Assume that for each $\widehat{\alpha i} \in$ A there exists a continuous function $\gamma_{\alpha \hat{i}}: I_{f, \widehat{\alpha i}}^{\alpha} \rightarrow \mathbb{R}$ and an increasing homeomorphism $\beta_{\widehat{\alpha i}}: I_{f, \widehat{\alpha i}}^{\alpha} \rightarrow I_{g, \alpha i}^{\alpha}$ such that

$$
\mu_{f, \widehat{\alpha i}}(s)=\left(\mu_{g, \widehat{\alpha i}} \circ \beta_{\widehat{\alpha i}}\right)(s)+\gamma_{\alpha \widehat{\alpha} i}(s), \quad s \in I_{f, \alpha \widehat{\alpha i}}^{\alpha},
$$

satisfying $\lim _{s \rightarrow c_{f, \alpha \widehat{i}}^{\alpha}} \beta_{\widehat{\alpha i}}(s)=c_{g, \alpha \widehat{i}}^{\alpha}, \lim _{s \rightarrow c_{f, \widehat{\alpha i}}^{\alpha}} \gamma_{\widehat{\alpha i}}(s)=a_{g, \widehat{\alpha i}}^{\alpha}$ for some $a_{g, \widehat{\alpha i}}^{\alpha} \in$ $\mathbb{R}$, where $\mu_{f, \widehat{\alpha i}}, \mu_{g, \widehat{\alpha i}}, c_{f, \widehat{\alpha i}}^{\alpha}, d_{f, \widehat{\alpha i}}^{\alpha}, c_{g, \widehat{\alpha i}}^{\alpha}, d_{g, \widehat{\alpha i}}^{\alpha}$ are those occurring in Theorem 1.2 , and $I_{f, \widehat{\alpha i}}^{\alpha}:=\left(c_{f, \widehat{\alpha i}}^{\alpha}, c_{f, \widehat{\alpha i}}^{\alpha}+\varepsilon_{f, \widehat{\alpha i}}^{\alpha}\right), I_{g, \widehat{\alpha i}}^{\alpha}:=\left(c_{g, \widehat{\alpha i}}^{\alpha}, c_{g, \widehat{\alpha i}}^{\alpha}+\varepsilon_{f, \widehat{\alpha} \hat{i}}^{\alpha}\right)$ in case

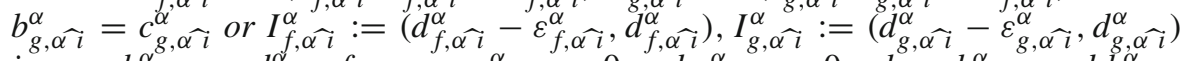
in case $b_{g, \widehat{\alpha i}}^{\alpha}=d_{g, \alpha \hat{i}}^{\alpha}$ for some $\varepsilon_{f, \alpha i}^{\alpha}>0$ and $\varepsilon_{g, \widehat{\alpha i}}^{\alpha}>0$, where $b_{f, \alpha \widehat{\alpha}}^{\alpha}$ and $b_{g, \alpha i}^{\alpha}$ are defined by (10) and (11). Moreover, we assume that for $\widehat{\alpha i}, \widehat{\alpha j} \in A, i \neq j$ we have $\beta_{\widehat{\alpha i}}=\beta_{\widehat{\alpha j} j}$ in the case where $C_{f, \widehat{\alpha i}}^{\alpha}=C_{f, \widehat{\alpha j}}^{\alpha}$ and $C_{f, \widehat{\alpha i}}, C_{f, \widehat{\alpha j}}$ are contained in the same component of $\mathbb{R} \backslash C_{f, \hat{\alpha} \hat{i}}^{\alpha}$. Then the flows $\left\{f^{t}: t \in \mathbb{R}\right\}$ and $\left\{g^{t}: t \in \mathbb{R}\right\}$ are topologically conjugate.

Proof On account of Theorem 2.4 we can take the same set of indices $A$ for the flows $\left\{f^{t}: t \in \mathbb{R}\right\}$ and $\left\{g^{t}: t \in \mathbb{R}\right\}$. Let $\left\{C_{f, \alpha}: \alpha \in A\right\},\left\{U_{f, \alpha}: \alpha \in A\right\},\left\{\varphi_{f, \alpha}: \alpha \in A\right\}$ and 
$\left\{C_{g, \alpha}: \alpha \in A\right\},\left\{U_{g, \alpha}: \alpha \in A\right\},\left\{\varphi_{g, \alpha}: \alpha \in A\right\}$ be the families described before the statement of this theorem. Then $C_{f, \alpha}=\varphi_{f, \alpha}^{-1}(\mathbb{R} \times\{0\})$ and $C_{g, \alpha}=\varphi_{g, \alpha}^{-1}(\mathbb{R} \times\{0\})$. Let $K_{f, \alpha}:=\varphi_{f, \alpha}^{-1}(\{0\} \times \mathbb{R})$ and $K_{g, \alpha}:=\varphi_{g, \alpha}^{-1}(\{0\} \times \mathbb{R})$ for $\alpha \in A$.

For all $\alpha \in A$ put $G_{f, \alpha}:=\varphi_{f, \alpha}^{-1}(\mathbb{R} \times[0,+\infty))$ if $\alpha \in A^{+}$and $G_{f, \alpha}:=\varphi_{f, \alpha}^{-1}(\mathbb{R} \times$ $(-\infty, 0])$ if $\alpha \in A^{-}$. By $H_{f, \alpha}$ denote the component of $\mathbb{R}^{2} \backslash C_{f, \alpha}$ which contains $G_{f, \alpha}$. In particular, we have $C_{f, \widehat{\alpha i}} \subset H_{f, \alpha}$ for all $\widehat{\alpha i} \in A$. Similarly, let $G_{g, \alpha}:=$ $\varphi_{g, \alpha}^{-1}(\mathbb{R} \times[0,+\infty))$ if $\alpha \in A^{+}$and $G_{g, \alpha}:=\varphi_{g, \alpha}^{-1}(\mathbb{R} \times(-\infty, 0])$ if $\alpha \in A^{-}$. By $H_{g, \alpha}$ denote the component of $\mathbb{R}^{2} \backslash C_{g, \alpha}$ which contains $G_{g, \alpha}$. Then $C_{g, \alpha i} \subset H_{g, \alpha}$ for all $\widehat{\alpha \lambda} \in A$.

For every positive integer $n$ let us put $A_{n}^{+}:=\left\{\alpha \in A^{+}:|\alpha|=n\right\}$ and $A_{n}^{-}:=\{\alpha \in$ $\left.A^{-}:|\alpha|=n\right\}$, where $|\alpha|$ denotes the length of the sequence $\alpha$. Then by the definition of an admissible class of finite sequences we get that if $A_{n}^{+}=\varnothing$ for some $n$, then $A_{m}^{+}=\emptyset$ for all $m>n$. Similarly, if $A_{n}^{-}=\emptyset$ for some $n$, then $A_{m}^{-}=\emptyset$ for all $m>n$.

For each positive integer $n$ such that $A_{n}^{+} \neq \varnothing$ we define $U_{f, n}^{+}$by taking $U_{f, n}^{+}:=G_{f, 1}$ in case $n=1$ and $U_{f, n}^{+}:=U_{f, n-1}^{+} \cup \bigcup_{\alpha \in A_{n}^{+}} G_{f, \alpha}$ in case $n>1$. Similarly, if $A_{n}^{-} \neq \emptyset$, then we put $U_{f, n}^{-}:=G_{f,-1}$ in case $n=1$ and $U_{f, n}^{-}:=U_{f, n-1}^{-} \cup \cup_{\alpha \in A_{n}^{-}} G_{f, \alpha}$ in case $n>1$. In the same way we define the regions $U_{g, n}^{+}$and $U_{g, n}^{-}$.

Let $\Phi: \mathbb{R}^{2} \rightarrow \mathbb{R}^{2}$ be a homeomorphism which realizes the topological equivalence. Then

$$
b_{f, \widehat{\alpha i}}^{\alpha}<b_{f, \widehat{\alpha} j}^{\alpha} \quad \text { iff } \quad b_{g, \widehat{\alpha i}}^{\alpha}<b_{g, \widehat{\alpha} j}^{\alpha}
$$

for all $\widehat{\alpha i}, \widehat{\alpha j} \in A, i \neq j$, since for each $\alpha \in A$ the homeomorphism $\varphi_{g, \alpha} \circ \Phi \circ \varphi_{f, \alpha}^{-1}$ is strictly increasing with respect to the second variable.

Let us start the construction of a homeomorphism which conjugates the flows $\left\{f^{t}: t \in \mathbb{R}\right\}$ and $\left\{g^{t}: t \in \mathbb{R}\right\}$. First, we consider the regions $U_{f, 1}:=U_{f, 1}^{+} \cup U_{f, 1}^{-}$ and $U_{g, 1}:=U_{g, 1}^{+} \cup U_{g, 1}^{-}$. Let us note that there exists a topological conjugacy $\Psi_{1}$ : $U_{f, 1} \rightarrow U_{g, 1}$ of flows $\left\{\left.f^{t}\right|_{U_{f, 1}}: t \in \mathbb{R}\right\}$ and $\left\{\left.g^{t}\right|_{U_{g, 1}}: t \in \mathbb{R}\right\}$, since $U_{f, 1}$ and $U_{g, 1}$ are parallelizable regions. Let us remind that $C_{f, 1}=C_{f,-1}, U_{f, 1}=U_{f,-1}, \varphi_{f, 1}=\varphi_{f,-1}$ and $C_{g, 1}=C_{g,-1}, U_{g, 1}=U_{g,-1}, \varphi_{g, 1}=\varphi_{g,-1}$. Thus, we can take $\Psi_{-1}=\Psi_{1}$. Moreover, we can choose $\Psi_{1}$ to satisfy the condition $\Psi_{1}\left(C_{f, 1}\right)=C_{g, 1}$ and $\Psi_{1}\left(K_{f, 1}\right)=$ $K_{g, 1}$. Without loss of generality we can assume that $A_{2}^{+} \neq \emptyset$ and $A_{2}^{-} \neq \emptyset$. Otherwise, we do need no other conditions on $\Psi_{1}$ on $G_{f, 1}$ or $G_{f,-1}$. Since $\Psi_{-1}=\Psi_{1}$, we also will consider sequences $-\widehat{\Gamma j} \in A$ for $\Psi_{1}$.

We can choose $\Psi_{1}$ in such a way that $\Psi_{1}\left(C_{f, 1 \widehat{i}}^{1}\right)=C_{g, 1 \uparrow}^{1}$ and $\Psi_{1}\left(C_{f,-\Upsilon_{j}}^{-1}\right)=$ $C_{g,-\Upsilon_{j}}^{-1}$, i.e., condition (9) holds for $\Psi_{1}$ and $\widehat{\Upsilon} \hat{i},-\widehat{\Upsilon} \hat{j} \in A$. (This condition is required for the existence of an extension of $\Psi_{1}$ to a topological conjugacy of flows $\left\{f^{t}: t \in\right.$ $\mathbb{R}\}$ and $\left\{g^{t}: t \in \mathbb{R}\right\}$.) More precisely, since $\varphi_{f, 1}: U_{f, 1} \rightarrow \mathbb{R}^{2}$ and $\varphi_{g, 1}: U_{g, 1} \rightarrow$ $\mathbb{R}^{2}$ are parallelizing homeomorphisms for $\left\{\left.f^{t}\right|_{U_{f, 1}}: t \in \mathbb{R}\right\}$ and $\left\{\left.g^{t}\right|_{U_{g, 1}}: t \in \mathbb{R}\right\}$, respectively, we can put $\Psi_{1}(p):=\left(\varphi_{g, 1}^{-1} \circ \psi_{1} \circ \varphi_{f, 1}\right)(p)$ for $p \in U_{f, 1}$, where $\psi_{1}$ : $\mathbb{R}^{2} \rightarrow \mathbb{R}^{2}, \psi_{1}(t, s):=\left(t, \theta_{1}(s)\right)$ and $\theta_{1}: \mathbb{R} \rightarrow \mathbb{R}$ is a homeomorphism such that $\theta_{1}(0)=0, \theta_{1}\left(b_{f, \Upsilon_{i}}^{1}\right)=b_{g, \Upsilon_{i}}^{1}, \theta_{1}\left(b_{f,-\uparrow_{j}}^{-1}\right)=b_{g,-\uparrow_{j}}^{-1}$ and $\theta_{1}$ restricted to $I_{f, \Upsilon_{i}}^{1}$ and $I_{f,-\uparrow j}^{-1}$ is equal to $\beta_{\uparrow i}$ and $\beta_{-\uparrow}$, respectively, for all $\widehat{1} i,-\Upsilon_{j} \in A$. The existence 
of such $\theta_{1}$ is guaranteed by condition (14). Let us note that $\Psi_{1}\left(K_{f, 1}\right)=K_{g, 1}$, since $\psi_{1}(0, s)=\left(0, \theta_{1}(s)\right)$.

Fix a positive integer $n$ such that $A_{n+1}^{+} \neq \emptyset$. Assume that we have defined a homeomorphism $\Psi_{n}: U_{f, n}^{+} \rightarrow U_{g, n}^{+}$which conjugates the flows $\left\{\left.f^{t}\right|_{U_{f, n}^{+}}: t \in \mathbb{R}\right\}$ and $\left\{\left.g^{t}\right|_{U_{g, n}^{+}}: t \in \mathbb{R}\right\}$ in such a way such that for each $\widehat{\alpha i} \in A^{+}$with $|\alpha| \leq n$ we have $\Psi_{n}\left(C_{f, \alpha}\right)=C_{g, \alpha}$ and

$$
\Psi_{n}\left(C_{f, \alpha \widehat{\alpha i}}^{\alpha}\right)=C_{g, \alpha \widehat{\alpha i}}^{\alpha},
$$

i.e., condition (9) holds for $\Psi_{n}$. The homeomorphism $\Psi_{n}$ is obtained by means of $\Psi_{1}$ defined on $U_{f, 1}$ and $\Psi_{\alpha}$ defined on $G_{f, \widehat{\alpha} \widehat{i}}$. The function $\Psi_{1}$ has been defined in the first step. For $\alpha \in A^{+}$with $|\alpha| \leq n$ we have $\Psi_{\alpha}: G_{f, \alpha} \rightarrow G_{f, \alpha}$ given by the formula $\Psi_{\alpha}:=\varphi_{g, \alpha}^{-1} \circ \psi_{\alpha} \circ\left(\left.\varphi_{f, \alpha}\right|_{\left.G_{f, \alpha}\right\}}\right)$, where

$$
\psi_{\alpha}(t, s)=\left(\eta_{\alpha}+t, \theta_{\alpha}(s)\right), \quad(t, s) \in \mathbb{R} \times[0,+\infty),
$$

and $\theta_{\alpha}:[0,+\infty) \rightarrow[0,+\infty)$ is an increasing homeomorphism. In case $A_{n+1}^{+} \neq \emptyset$ we have $\theta_{\alpha}\left(b_{f, \widehat{\alpha i}}^{\alpha}\right)=b_{g, \alpha \widehat{\alpha i}}^{\alpha}$ and $\theta_{\alpha}$ restricted to $I_{f, \widehat{\alpha i}}^{\alpha}$ is equal to $\beta_{\alpha \widehat{\imath i}}$. By the construction of $\Psi_{1}$ we have $\eta_{1}=0$, since $\Psi_{1}\left(K_{f, 1}\right)=K_{g, 1}$. The numbers $\eta_{\alpha}$ are constructed in the subsequent steps.

Let us fix any $\widehat{\alpha i} \in A_{n+1}^{+}$. We will define $\Psi_{\widehat{\alpha i}}: G_{f, \widehat{\alpha i}} \rightarrow G_{g, \widehat{\alpha i}}$ and use it to construct a homeomorphism $\Psi_{n+1}: U_{f, n+1}^{+} \rightarrow U_{g, n+1}^{+}$which conjugates the flows $\left\{\left.f^{t}\right|_{U_{f, n+1}^{+}}: t \in \mathbb{R}\right\}$ and $\left\{\left.g^{t}\right|_{U_{g, n+1}^{+}}: t \in \mathbb{R}\right\}$. The first step is to define $\Psi_{\widehat{\alpha i}}$ on $C_{f, \widehat{\alpha i}}$. To do this we use the form of the function $\Psi_{\alpha}: G_{f, \alpha} \rightarrow G_{g, \alpha}$ used in the construction of $\Psi_{n}: U_{f, n}^{+} \rightarrow U_{g, n}^{+}$.

Denote by $(t, s)$ the coordinates of points belonging to $U_{f, \alpha}$ in the chart $\varphi_{f, \alpha}$ and by $\left(t^{\prime}, s^{\prime}\right)$ the coordinates of points belonging to $U_{f, \widehat{\alpha i}}$ in the chart $\varphi_{f, \widehat{\alpha i}}$. Then by (8) we have $t^{\prime}=\mu_{f, \widehat{\alpha i}}(s)+t$ and $s^{\prime}=v_{f, \widehat{\alpha i}}(s)$ for $t \in \mathbb{R}$ and $s \in\left(c_{f, \alpha \widehat{\alpha}}^{\alpha}, d_{f, \alpha \widehat{i}}^{\alpha}\right)$. Similarly, denote by $(u, v)$ the coordinates of points belonging to $U_{g, \alpha}$ in the chart $\varphi_{g, \alpha}$ and by $\left(u^{\prime}, v^{\prime}\right)$ the coordinates of points belonging to $U_{g, \widehat{\alpha i}}$ in the chart $\varphi_{g, \widehat{\alpha i}}$. Then $u^{\prime}=\mu_{g, \widehat{\alpha i}}(v)+u$ and $v^{\prime}=v_{g, \alpha \hat{i}}(v)$ for $u \in \mathbb{R}$ and $v \in\left(c_{g, \alpha \widehat{i}}^{\alpha}, d_{g, \alpha \widehat{i}}^{\alpha}\right)$.

Let us recall that $\psi_{\alpha}=\varphi_{g, \alpha} \circ \Psi_{\alpha} \circ\left(\left.\varphi_{f, \alpha}\right|_{G_{f, \alpha}}\right)^{-1}$ and

$$
\psi_{\alpha}(t, s)=\left(\eta_{\alpha}+t, \theta_{\alpha}(s)\right), \quad(t, s) \in \mathbb{R} \times[0,+\infty),
$$

where $\eta_{\alpha} \in \mathbb{R}$ and $\theta_{\alpha}:[0,+\infty) \rightarrow[0,+\infty)$ is an increasing homeomorphism such that $\theta_{\alpha}\left(b_{f, \alpha \widehat{\alpha i}}^{\alpha}\right)=b_{g, \alpha \widehat{\alpha i}}^{\alpha}$ and $\theta_{\alpha}$ restricted to $I_{f, \alpha \widehat{\alpha i}}^{\alpha}$ is equal to $\beta_{\alpha \widehat{i}}$. Passing to coordinates we have $u=\eta_{\alpha}+t$ and $v=\theta_{\alpha}(s)$ for $t \in \mathbb{R}$ and $s \in[0,+\infty)$ if $(u, v)=\psi_{\alpha}(t, s)$.

Consider the case where $b_{f, \widehat{\alpha i}}^{\alpha}=c_{f, \alpha \widehat{i}}^{\alpha}$. (The case $b_{f, \alpha \widehat{\alpha}}^{\alpha}=d_{f, \alpha \widehat{i}}^{\alpha}$ is similar.) Then $b_{g, \alpha \hat{i}}^{\alpha}=c_{g, \widehat{\alpha} \hat{i}}^{\alpha}$, since $\left\{f^{t}: t \in \mathbb{R}\right\}$ and $\left\{g^{t}: t \in \mathbb{R}\right\}$ are topologically equivalent. From the assumption we have that there exist a continuous function $\gamma_{\widehat{\alpha i}}: I_{f, \alpha \widehat{\alpha}}^{\alpha} \rightarrow \mathbb{R}$ and an increasing homeomorphism $\beta_{\widehat{\alpha i} i}: I_{f, \widehat{\alpha i}}^{\alpha} \rightarrow I_{g, \widehat{\alpha i}}^{\alpha}$ such that

$$
\mu_{f, \widehat{\alpha i}}(s)=\left(\mu_{g, \widehat{\alpha i}} \circ \beta_{\widehat{\alpha i}}\right)(s)+\gamma_{\widehat{\alpha i}}(s), \quad s \in I_{f, \widehat{\alpha i}}^{\alpha},
$$


and $\lim _{s \rightarrow c_{f, \widehat{\alpha i}}^{\alpha}} \beta_{\widehat{\alpha i}}(s)=c_{g, \widehat{\alpha i}}^{\alpha}, \lim _{s \rightarrow c_{f, \widehat{\alpha i}}^{\alpha}} \gamma_{\widehat{\alpha} \widehat{i}}(s)=a_{g, \alpha \widehat{\alpha}}^{\alpha}$ for some $a_{g, \widehat{\alpha i}}^{\alpha} \in \mathbb{R}$.

By (8) we have $t^{\prime}=\mu_{f, \widehat{\alpha i}}(s)+t$ and $s^{\prime}=v_{f, \widehat{\alpha i}}(s)$ for $t \in \mathbb{R}$ and $s \in$ $\left(c_{f, \widehat{\alpha i}}^{\alpha}, d_{f, \widehat{\alpha i}}^{\alpha}\right)$. Hence, $t=t^{\prime}-\mu_{f, \widehat{\alpha i}}\left(v_{f, \alpha \widehat{i}}^{-1}\left(s^{\prime}\right)\right)$ and $s=v_{f, \alpha \widehat{\alpha}}^{-1}\left(s^{\prime}\right)$. By the definition of the $\psi_{\alpha}$, if $(u, v)=\psi_{\alpha}(t, s)$, then $u=\eta_{\alpha}+t$ and $v=\beta_{\alpha \hat{i}}(s)$ for $t \in \mathbb{R}$ and $s \in\left(c_{f, \widehat{\alpha} \hat{i}}^{\alpha}, c_{f, \widehat{\alpha i}}^{\alpha}+\varepsilon_{f, \widehat{\alpha i}}^{\alpha}\right)$. (In case $b_{f, \alpha \widehat{i}}^{\alpha}=d_{f, \alpha \widehat{i}}^{\alpha}$ we would have $\left.s \in\left(d_{f, \widehat{\alpha i}}^{\alpha}-\varepsilon_{f, \widehat{\alpha i}}^{\alpha}, d_{f, \alpha \hat{\alpha}}^{\alpha}\right).\right)$

Put $d_{f, \widehat{\alpha i}}:=v_{f, \widehat{\alpha i}}\left(c_{f, \widehat{\alpha i}}^{\alpha}+\varepsilon_{f, \widehat{\alpha i}}^{\alpha}\right)$. Then for the function $h_{f, \widehat{\alpha i}}$ given by (7) we have $\left.h_{f, \widehat{\alpha i}}\left(\mathbb{R} \times I_{f, \widehat{\alpha} \widehat{i}}^{\alpha}\right)=\mathbb{R} \times\left(d_{f, \widehat{\alpha i}}, 0\right)\right)$. Put $W_{f, \widehat{\alpha i}}:=\varphi_{f, \widehat{\alpha i}}^{-1}\left(\mathbb{R} \times\left(d_{f, \widehat{\alpha i}}, 0\right)\right)$. Let $\phi_{\widehat{\alpha} \hat{i}}^{\alpha}\left(t^{\prime}, s^{\prime}\right):=\left(\varphi_{g, \widehat{\alpha i}} \circ \Psi_{\alpha} \circ \varphi_{f, \widehat{\alpha} \widehat{i}}^{-1}\right)\left(t^{\prime}, s^{\prime}\right)$ for $\left(t^{\prime}, s^{\prime}\right) \in \mathbb{R} \times\left(d_{f, \widehat{\alpha i}}, 0\right)$. Since $u^{\prime}=\mu_{g, \alpha i}(v)+u$ and $v^{\prime}=v_{g, \widehat{\alpha i}}(v)$, we have $u^{\prime}=\mu_{g, \widehat{\alpha i}}\left(\beta_{\alpha \widehat{\alpha i}}(s)\right)+\eta_{\alpha}+$ $t$ and $v^{\prime}=v_{g, \widehat{\alpha i}}\left(\beta_{\alpha \widehat{\alpha}}(s)\right)$. Hence, $u^{\prime}=\mu_{g, \widehat{\alpha i}}\left(\beta_{\alpha \widehat{\alpha i}}\left(v_{f, \widehat{\alpha} \hat{i}}^{-1}\left(s^{\prime}\right)\right)\right)+\eta_{\alpha}+t^{\prime}-$ $\mu_{f, \widehat{\alpha i}}\left(v_{f, \widehat{\alpha i}}^{-1}\left(s^{\prime}\right)\right)$ and $v^{\prime}=v_{g, \alpha \hat{i}}\left(\beta_{\alpha \widehat{\alpha}}\left(v_{f, \widehat{\alpha i}}^{-1}\left(s^{\prime}\right)\right)\right)$. Thus, by the assumption, we have $u^{\prime}=-\gamma_{\alpha \widehat{i}}\left(v_{f, \alpha \widehat{i}}^{-1}\left(s^{\prime}\right)\right)+\eta_{\alpha}+t^{\prime}$.

Put

$$
\eta_{\widehat{\alpha i}}\left(s^{\prime}\right):=-\gamma_{\alpha \widehat{i}}\left(v_{f, \widehat{\alpha i}}^{-1}\left(s^{\prime}\right)\right)+\eta_{\alpha}
$$

and

$$
\xi_{\alpha \widehat{\alpha}}\left(s^{\prime}\right):=v_{g, \widehat{\alpha i}}\left(\beta_{\alpha \widehat{\alpha i}}\left(v_{f, \widehat{\alpha i}}^{-1}\left(s^{\prime}\right)\right)\right)
$$

for $s^{\prime} \in\left(d_{f, \widehat{\alpha i}}, 0\right)$. Then

$$
\phi_{\alpha \widehat{\alpha} i}^{\alpha}\left(t^{\prime}, s^{\prime}\right):=\left(\eta_{\alpha \widehat{i}}\left(s^{\prime}\right)+t^{\prime}, \xi_{\alpha \widehat{\alpha i}}\left(s^{\prime}\right)\right), \quad\left(t^{\prime}, s^{\prime}\right) \in \mathbb{R} \times\left(d_{f, \widehat{\alpha i}}, 0\right) .
$$

Since $\lim _{s^{\prime} \rightarrow 0} \eta_{\widehat{\alpha i}}\left(s^{\prime}\right)=-a_{g, \widehat{\alpha i}}^{\alpha}+\eta_{\alpha}$ and $\lim _{s^{\prime} \rightarrow 0} \xi_{\widehat{\alpha i}}\left(s^{\prime}\right)=0$, we can extend continuously the functions $\eta_{\widehat{\alpha i}}$ and $\xi_{\alpha \widehat{\alpha i}}$ onto $\left(d_{f, \widehat{\alpha i}}, 0\right]$ by putting $\eta_{\widehat{\alpha i}}(0):=-a_{g, \widehat{\alpha i}}^{\alpha}+$ $\eta_{\alpha}$ and $\xi_{\alpha \widehat{i}}(0):=0$. The value $\eta_{\widehat{\alpha i}}(0)$ we will denote by $\eta_{\alpha \widehat{i}}$. Thus, we have obtained a continuous extension of $\Psi_{\alpha}$ on $C_{f, \hat{\alpha} i}$.

Now define $\Psi_{\alpha \widehat{i}}: G_{f, \widehat{\alpha i}} \rightarrow G_{f, \widehat{\alpha i}}$ by the formula $\Psi_{\alpha \widehat{i}}:=\varphi_{g, \widehat{\alpha i}}^{-1} \circ \psi_{\alpha \widehat{\alpha}} \circ$ $\left(\left.\varphi_{f, \widehat{\alpha i}}\right|_{G_{f, \widehat{\alpha i}}}\right)$, where

$$
\psi_{\widehat{\alpha i}}(t, s)=\left(\eta_{\alpha \widehat{i}}+t, \theta_{\alpha \widehat{\alpha} i}(s)\right), \quad(t, s) \in \mathbb{R} \times[0,+\infty),
$$

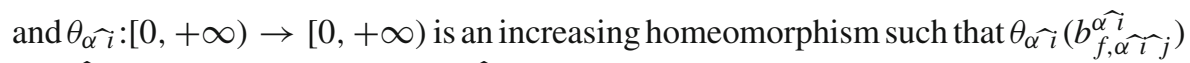

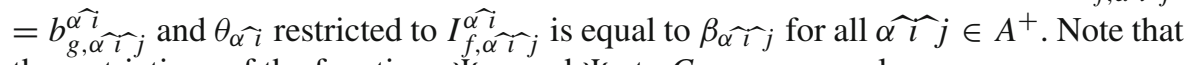
the restrictions of the functions $\Psi_{\widehat{\alpha i}}$ and $\Psi_{\alpha}$ to $C_{f, \widehat{\alpha i}}$ are equal.

Therefore, we can define a homeomorphism $\Psi_{n+1}: U_{f, n+1}^{+} \rightarrow U_{g, n+1}^{+}$which conjugates the flows $\left\{\left.f^{t}\right|_{U_{f, n+1}^{+}}: t \in \mathbb{R}\right\}$ and $\left\{\left.g^{t}\right|_{U_{g, n+1}^{+}}: t \in \mathbb{R}\right\}$ by

$$
\Psi_{n+1}(p):=\left\{\begin{array}{l}
\Psi_{n}(p) \text { if } p \in U_{f, n}^{+}, \\
\Psi_{\widehat{\alpha i} i}(p) \quad \text { if } p \in G_{f, \widehat{\alpha i}}, \widehat{\alpha i} \in A_{n+1}^{+} .
\end{array}\right.
$$

Then $\Psi_{n+1}$ conjugates the flows $\left\{\left.f^{t}\right|_{U_{f, n+1}^{+}}: t \in \mathbb{R}\right\}$ and $\left\{\left.g^{t}\right|_{U_{g, n+1}^{+}}: t \in \mathbb{R}\right\}$. 
In the same way we can extend $\Psi_{n}$ from $U_{f, n}^{-}$to $\Psi_{n+1}$ defined on $U_{f, n+1}^{-}$. Let $U_{f, n}:=U_{f, n}^{+} \cup U_{f, n}^{-}$. To define a homeomorphism $\Psi$ which realizes the topological conjugacy between the flows $\left\{f^{t}: t \in \mathbb{R}\right\}$ and $\left\{g^{t}: t \in \mathbb{R}\right\}$ we put

$$
\Psi(p):=\Psi_{n}(p), \quad p \in U_{f, n}, n \in \mathbb{Z}_{+} .
$$

The function $\Psi$ is well defined, since for $k>n$ we have $U_{f, n}^{+} \subset U_{f, k}^{+}$and $\left.\Psi_{k}\right|_{U_{f, n}^{+}}=$ $\left.\Psi_{n+1}\right|_{U_{f, n}^{+}}$.

Now we prove that the assumption of Theorem 3.2 concerning the relation between the functions $\mu_{f, \widehat{\alpha i}}$ and $\mu_{f, \widehat{\alpha i}}$ is a necessary condition for the topological conjugacy of Brouwer flows.

Proposition 3.3 Let $\left\{f^{t}: t \in \mathbb{R}\right\}$ and $\left\{g^{t}: t \in \mathbb{R}\right\}$ be topologically conjugate Brouwer flows. Then for each $\widehat{\alpha i} \in A$ there exist a continuous function $\gamma_{\alpha i}: I_{f, \alpha i}^{\alpha} \rightarrow \mathbb{R}$ and an increasing homeomorphism $\beta_{\widehat{\alpha i}}: I_{f, \alpha \widehat{i}}^{\alpha} \rightarrow I_{g, \alpha \widehat{i}}^{\alpha}$ such that relation (13) holds, i.e.,

$$
\mu_{f, \widehat{\alpha i}}(s)=\left(\mu_{g, \widehat{\alpha i}} \circ \beta_{\alpha \widehat{i}}\right)(s)+\gamma_{\alpha \widehat{i}}(s), \quad s \in I_{f, \widehat{\alpha i}}^{\alpha},
$$

where $\mu_{f, \widehat{\alpha i}}, \mu_{g, \widehat{\alpha i}}, c_{f, \widehat{\alpha i}}^{\alpha}, d_{f, \widehat{\alpha i}}^{\alpha}, c_{g, \widehat{\alpha i}}^{\alpha}, d_{g, \alpha \widehat{\alpha}}^{\alpha}$ are those occurring in Theorem 1.2, and $I_{f, \alpha i}^{\alpha}:=\left(c_{f, \alpha \widehat{i}}^{\alpha}, c_{f, \widehat{\alpha i}}^{\alpha}+\varepsilon_{f, \widehat{\alpha} \vec{i}}^{\alpha}\right), I_{g, \widehat{\alpha i}}^{\alpha}:=\left(c_{g, \widehat{\alpha i}}^{\alpha}, c_{g, \alpha \widehat{\alpha}}^{\alpha}+\varepsilon_{f, \widehat{\alpha i}}^{\alpha}\right)$ in case $b_{g, \widehat{\alpha i}}^{\alpha}=$ $c_{g, \widehat{\alpha i}}^{\alpha}$ or $I_{f, \widehat{\alpha i}}^{\alpha}:=\left(d_{f, \widehat{\alpha i}}^{\alpha}-\varepsilon_{f, \alpha \widehat{\alpha}}^{\alpha}, d_{f, \widehat{\alpha i}}^{\alpha}\right), I_{g, \widehat{\alpha i}}^{\alpha}:=\left(d_{g, \widehat{\alpha i}}^{\alpha}-\varepsilon_{g, \widehat{\alpha i}}^{\alpha}, d_{g, \widehat{\alpha i}}^{\alpha}\right)$ in case

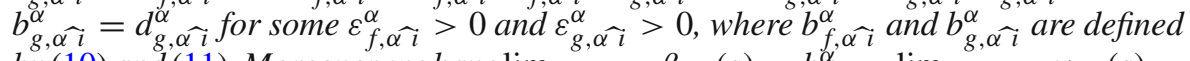
by (10) and (11). Moreover, we have $\lim _{s \rightarrow b_{f, \widehat{\alpha i}}^{\alpha}} \beta_{\widehat{\alpha i}}(s)=b_{g, \alpha \widehat{i}}^{\alpha}, \lim _{s \rightarrow b_{f, \widehat{\alpha i}}^{\alpha}} \gamma_{\widehat{\alpha i} i}(s)=$ $a_{g, \widehat{\alpha i}}^{\alpha}$ for some $a_{g, \widehat{\alpha i}}^{\alpha} \in \mathbb{R}$.

Proof Let $\left\{C_{f, \alpha}: \alpha \in A\right\},\left\{U_{f, \alpha}: \alpha \in A\right\},\left\{\varphi_{f, \alpha}: \alpha \in A\right\}$ and $\left\{C_{g, \alpha}: \alpha \in\right.$ $A\},\left\{U_{g, \alpha}: \alpha \in A\right\},\left\{\varphi_{g, \alpha}: \alpha \in A\right\}$ be the families described before the statement of Theorem 3.2. Let $\Phi: \mathbb{R}^{2} \rightarrow \mathbb{R}^{2}$ be a homeomorphism which realizes the topological conjugacy. Let us fix an arbitrary $\widehat{\alpha i} \in A$. We will consider the case where $b_{g, \alpha \hat{i}}^{\alpha}=$ $c_{g, \alpha \widehat{\alpha}}^{\alpha}$. (The second case is similar.)

By Theorem 1.2 we have $U_{f, \alpha} \cap U_{f, \widehat{\alpha i}}=\varphi_{f, \alpha}^{-1}\left(\mathbb{R} \times\left(c_{f, \widehat{\alpha i}}^{\alpha}, d_{f, \widehat{\alpha} \widehat{\alpha}}^{\alpha}\right)\right)$ and $U_{g, \alpha} \cap$ $U_{g, \widehat{\alpha i}}=\varphi_{g, \alpha}^{-1}\left(\mathbb{R} \times\left(c_{g, \widehat{\alpha i}}^{\alpha}, d_{g, \widehat{\alpha i}}^{\alpha}\right)\right)$. As in the proof of Theorem 3.2 we can find an $\varepsilon_{f, \widehat{\alpha i}}^{\alpha}>0$ such that the interval $\left(c_{f, \widehat{\alpha i}}^{\alpha}, c_{f, \widehat{\alpha i}}^{\alpha}+\varepsilon_{f, \widehat{\alpha i}}^{\alpha}\right)$ does not contain any $b_{f, \widehat{\alpha i} j}^{\alpha}$ for $j \neq i$. Put $C_{f, \varepsilon_{\tilde{\alpha} \vec{i}}}:=\varphi_{f, \alpha}^{-1}\left(\mathbb{R} \times\left\{c_{f, \widehat{\alpha} \widehat{i}}^{\alpha}+\varepsilon_{f, \widehat{\alpha} \widehat{i}}^{\alpha}\right\}\right)$ and $C_{g, \varepsilon_{\widehat{\alpha} \hat{i}}}:=\Phi\left(C_{f, \varepsilon_{\widehat{\alpha} \widehat{i}}}\right)$. Since $\Phi: \mathbb{R}^{2} \rightarrow \mathbb{R}^{2}$ is a homeomorphism which realizes the topological conjugacy, we get that there exists a unique $\varepsilon_{g, \alpha \widehat{\alpha i}}^{\alpha}>0$ such that interval $\left(c_{g, \alpha \widehat{\alpha}}^{\alpha}, c_{g, \alpha \widehat{\alpha}}^{\alpha}+\varepsilon_{g, \alpha \widehat{\alpha i}}^{\alpha}\right)$ does not contain any $b_{g, \widehat{\alpha} \hat{j}}^{\alpha}$ for $j \neq i$ and $C_{g, \varepsilon_{\widehat{\alpha i}}}=\varphi_{g, \alpha}^{-1}\left(\mathbb{R} \times\left\{c_{g, \widehat{\alpha i}}^{\alpha}+\varepsilon_{g, \widehat{\alpha i}}^{\alpha}\right\}\right)$. Denote by $\phi_{\widehat{\alpha i}}^{\alpha}$ the restriction of the function $\varphi_{g, \alpha} \circ \Phi \circ \varphi_{f, \alpha}^{-1}$ to the set $\mathbb{R} \times\left(c_{f, \widehat{\alpha} \widehat{i}}^{\alpha}, c_{f, \alpha \vec{\alpha}}^{\alpha}+\varepsilon_{f, \widehat{\alpha i}}^{\alpha}\right)$.

Then $\phi_{\alpha \widehat{\alpha} i}^{\alpha}: \mathbb{R} \times\left(c_{f, \alpha \hat{i}}^{\alpha}, c_{f, \widehat{\alpha i}}^{\alpha}+\varepsilon_{f, \alpha \hat{i}}^{\alpha}\right) \rightarrow \mathbb{R} \times\left(c_{\left\{g^{t}\right\}, \alpha \widehat{i}}^{\alpha}, c_{g, \widehat{\alpha i}}^{\alpha}+\varepsilon_{g, \widehat{\alpha i}}^{\alpha}\right)$. Thus, if $(u, v)=\psi_{\widehat{\alpha i}}^{\alpha}(t, s)$, then $v=\beta_{\widehat{\alpha i}}(s)$, where $\beta_{\widehat{\alpha i}}:\left(c_{f, \widehat{\alpha i}}^{\alpha}, c_{f, \widehat{\alpha i}}^{\alpha}+\varepsilon_{f, \widehat{\alpha i}}^{\alpha}\right) \rightarrow$ $\left(c_{g, \alpha \widehat{i}}^{\alpha}, c_{g, \alpha \widehat{i}}^{\alpha}+\varepsilon_{g, \alpha \widehat{i}}^{\alpha}\right)$ is an increasing homeomorphism.

Let $K_{f, \alpha}:=\varphi_{f, \alpha}^{-1}(\{0\} \times \mathbb{R})$ and $K_{f, \widehat{\alpha} i}:=\varphi_{f, \alpha \widehat{i}}^{-1}(\{0\} \times \mathbb{R})$. Then $\mu_{f, \alpha \widehat{i}}(s)$ describes the time needed for the flow $\left\{f^{t}: t \in \mathbb{R}\right\}$ to move along the trajectory $\varphi_{f, \alpha}^{-1}(\mathbb{R} \times\{s\})$ 
from the section $K_{f, \alpha \widehat{i}}$ to the section $K_{f, \alpha}$ for each $s \in\left(c_{f, \alpha \widehat{\alpha},}^{\alpha}, c_{f, \alpha \widehat{\alpha}}^{\alpha}+\varepsilon_{f, \alpha \widehat{\alpha i}}^{\alpha}\right)$. Put $L_{g, \alpha}:=\Phi\left(K_{f, \alpha}\right)$ and $L_{g, \widehat{\alpha i}}:=\Phi\left(K_{f, \alpha \hat{i}}\right)$. Then $L_{g, \alpha}$ is a section in $U_{g, \alpha}$ and $L_{g, \widehat{\alpha i} \hat{i}}$ is a section in $U_{g, \widehat{\alpha i}}$, since $\Phi$ is a homeomorphism which maps trajectories of $\left\{f^{t}: t \in \mathbb{R}\right\}$ onto trajectories of $\left\{g^{t}: t \in \mathbb{R}\right\}$. Moreover, by the assumption that $\Phi: \mathbb{R}^{2} \rightarrow \mathbb{R}^{2}$ realizes the topological conjugacy we get that $\mu_{f, \alpha} \widehat{i}(s)$ is equal to the time needed for the flow $\left\{g^{t}: t \in \mathbb{R}\right\}$ to move along the trajectory $\varphi_{g, \alpha}^{-1}\left(\mathbb{R} \times\left\{\beta_{\widehat{\alpha i}}(s)\right\}\right)$ from the section $L_{g, \widehat{\alpha i}}$ to the section $L_{g, \alpha}$ for each $s \in\left(c_{f, \alpha \widehat{\alpha}}^{\alpha}, c_{f, \widehat{\alpha i}}^{\alpha}+\varepsilon_{f, \alpha \widehat{i}}^{\alpha}\right)$.

Let $K_{g, \alpha}:=\varphi_{g, \alpha}^{-1}(\{0\} \times \mathbb{R})$ and $K_{g, \widehat{\alpha i}}:=\varphi_{g, \widehat{\alpha i}}^{-1}(\{0\} \times \mathbb{R})$. For every $s \in$ $\left(c_{f, \widehat{\alpha i}}^{\alpha}, c_{f, \alpha \widehat{\alpha}}^{\alpha}+\varepsilon_{f, \widehat{\alpha i}}^{\alpha}\right)$ denote by $\tau_{g, \alpha}(s)$ the time needed for the flow $\left\{g^{t}: t \in \mathbb{R}\right\}$ to move along the trajectory $\varphi_{g, \alpha}^{-1}\left(\mathbb{R} \times\left\{\beta_{\alpha \widehat{i}}(s)\right\}\right)$ from $K_{g, \alpha}$ to $L_{g, \alpha}$ and by $\tau_{g, \alpha \hat{\alpha}}(s)$ the time needed to move along this trajectory from $K_{g, \alpha \widehat{i}}$ to $L_{g, \alpha \widehat{i}}$. Then for each $s \in\left(c_{f, \hat{\alpha i} i}^{\alpha}, c_{f, \alpha \widehat{i}}^{\alpha}+\varepsilon_{f, \alpha \hat{i}}^{\alpha}\right)$ we have

$$
\mu_{g, \widehat{\alpha i}}\left(\beta_{\alpha \widehat{i}}(s)\right)=\mu_{f, \widehat{\alpha i}}(s)+\tau_{\alpha \widehat{i}}(s)-\tau_{\alpha}(s) .
$$

Define $\gamma_{\alpha \widehat{i}}:\left(c_{f, \widehat{\alpha i}}^{\alpha}, c_{f, \widehat{\alpha i}}^{\alpha}+\varepsilon_{f, \widehat{\alpha i}}^{\alpha}\right) \rightarrow \mathbb{R}$ by putting

$$
\gamma_{\alpha \widehat{i} i}(s):=\tau_{\alpha}(s)-\tau_{\alpha \widehat{i}}(s), \quad s \in\left(c_{f, \widehat{\alpha i}}^{\alpha}, c_{f, \widehat{\alpha i}}^{\alpha}+\varepsilon_{f, \alpha \widehat{i}}^{\alpha}\right) .
$$

Then

$$
\mu_{f, \widehat{\alpha i}}(s)=\left(\mu_{g, \widehat{\alpha i}} \circ \beta_{\widehat{\alpha i}}\right)(s)+\gamma_{\alpha \widehat{\alpha i}}(s)
$$

for each $s \in\left(c_{f, \hat{\alpha i}}^{\alpha}, c_{f, \alpha \widehat{\alpha}}^{\alpha}+\varepsilon_{f, \hat{\alpha} \widehat{i}}^{\alpha}\right)$.

Since $C_{f, \widehat{\alpha i}}^{\alpha}=\varphi_{f, \alpha}^{-1}\left(\mathbb{R} \times\left\{c_{f, \widehat{\alpha i}}^{\alpha}\right\}\right), C_{g, \alpha \widehat{i}}^{\alpha}=\varphi_{g, \alpha}^{-1}\left(\mathbb{R} \times\left\{c_{g, \widehat{\alpha i}}^{\alpha}\right\}\right)$ and $\Phi\left(C_{f, \alpha \widehat{\alpha i}}^{\alpha}\right)=$ $C_{g, \alpha \widehat{i}}^{\alpha}$, we have $\lim _{s \rightarrow c_{f, \alpha \widehat{i}}^{\alpha}} \beta_{\widehat{\alpha i}}(s)=c_{g, \widehat{\alpha i}}^{\alpha}$. Moreover, by the definition of $\gamma_{\widehat{\alpha} \hat{i}}$ and $\tau_{\alpha}$

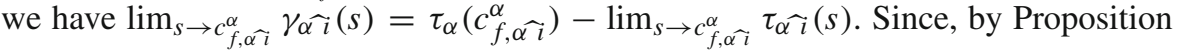
1.6, $v=c_{g, \alpha \widehat{i}}^{\alpha}$ corresponds to $v^{\prime}=0$, we get that $\lim _{s \rightarrow c_{f, \widehat{\alpha i}}^{\alpha}} \tau_{\widehat{\alpha} \hat{i}}(s)$ is the time needed to move along trajectory $C_{g, \widehat{\alpha i}}$ from $K_{g, \widehat{\alpha i}}$ to $L_{g, \widehat{\alpha i}}$. Thus, $\lim _{s \rightarrow c_{f, \widehat{\alpha} \hat{i}}^{\alpha}} \gamma_{\widehat{\alpha} \widehat{i}}(s)=a_{g, \widehat{\alpha} \hat{i}}^{\alpha}$ for some $a_{g, \alpha \widehat{\alpha}}^{\alpha} \in \mathbb{R}$.

Summing up, we proved that relation (13) is a sufficient and necessary condition for topologically equivalent Brouwer flows to be topologically conjugate.

Open Access This article is distributed under the terms of the Creative Commons Attribution 4.0 International License (http://creativecommons.org/licenses/by/4.0/), which permits unrestricted use, distribution, and reproduction in any medium, provided you give appropriate credit to the original author(s) and the source, provide a link to the Creative Commons license, and indicate if changes were made.

\section{References}

1. Andrea, S.A.: On homeomorphisms of the plane which have no fixed points. Abh. Math. Sem. Hamburg 30, 61-74 (1967)

2. Beck, A.: Continuous flows in the plane, Grundlehren der Mathematischen Wissenschaften 201. Springer (1974) 
3. Béguin, F., Le Roux, F.: Ensemble oscillant d'un homéomorphisme de Brouwer, homéomorphismes de Reeb. Bull. Soc. Math. France 131(2), 149-210 (2003)

4. Bhatia, N.P., Szegö, G.P.: Stability Theory of Dynamical Systems. Springer, Berlin (1970)

5. Brouwer, L.E.J.: Beweis des ebenen Translationssatzes. Math. Ann. 72, 37-54 (1912)

6. Dumortier, F., Llibre, J., Artés, J.C.: Qualitative Theory of Planar Differential Systems. Springer, Berlin (2006)

7. Hájek, O.: Dynamical Systems in the Plane. Academic Press, London (1968)

8. Homma, T., Terasaka, H.: On the structure of the plane translation of Brouwer. Osaka Math. J. 5, 233-266 (1953)

9. Irwin, M.C.: Smooth Dynamical Systems. Academic Press, London (1980)

10. Kaplan, W.: Regular curve-families filling the plane I. Duke Math. J. 7, 154-185 (1940)

11. Leśniak, Z.: On a decomposition of the plane for a flow free mappings. Publ. Math. Debrecen 75(1-2), 191-202 (2009)

12. Leśniak, Z.: On boundaries of parallelizable regions of flows of free mappings, Abstr. Appl. Anal. 2007 (2007), Article ID 31693

13. Leśniak, Z.: On strongly irregular points of a Brouwer homeomorphism embeddable in a flow, Abstr. Appl. Anal. 2014 (2014), Article ID 638784

14. Leśniak, Z.: On the first prolongational limit set of flows of free mappings. Tamkang J. Math. 39, 263-269 (2008)

15. Leśniak, Z:: On the structure of Brouwer homeomorphisms embeddable in a flow, Abstr. Appl. Anal. 2012 (2012), Article ID 248413

16. Leśniak, Z.: On the topological equivalence of flows of Brouwer homeomorphisms. J. Differ. Equ. Appl. 22, 853-864 (2016)

17. Le Roux, F.: Classes de conjugaison des flots du plan topologiquement équivalents au flot de Reeb. C. R. Acad. Sci. Paris 328(1), 45-50 (1999)

18. Matsumoto, S.: A characterization of the standard Reeb flow. Hokkaido Math. J. 42(1), 69-80 (2013)

19. McCann, R.C.: Planar dynamical systems without critical points. Funkcial. Ekvac. 13, 67-95 (1970)

20. Nakayama, H.: On dimensions of non-Hausdorff sets for plane homeomorphisms. J. Math. Soc. Jpn. 47(4), 789-793 (1995) 